TRANSACTIONS OF THE

AMERICAN MATHEMATICAL SOCIETY

Volume 352 , Number 10 , Pages 4731-4757

S 0002-9947(00)02591-5

Article electronically published on June 9, 2000

\title{
ENDOMORPHISMS OF EXPANSIVE SYSTEMS ON COMPACT METRIC SPACES AND THE PSEUDO-ORBIT TRACING PROPERTY
}

\author{
MASAKAZU NASU
}

\begin{abstract}
We investigate the interrelationships between the dynamical properties of commuting continuous maps of a compact metric space. Let $X$ be a compact metric space.

First we show the following. If $\tau: X \rightarrow X$ is an expansive onto continuous map with the pseudo-orbit tracing property (POTP) and if there is a topologically mixing continuous map $\varphi: X \rightarrow X$ with $\tau \varphi=\varphi \tau$, then $\tau$ is topologically mixing. If $\tau: X \rightarrow X$ and $\varphi: X \rightarrow X$ are commuting expansive onto continuous maps with POTP and if $\tau$ is topologically transitive with period $p$, then for some $k$ dividing $p, X=\bigcup_{i=0}^{l-1} B_{i}$, where the $B_{i}, 0 \leq i \leq l-1$, are the basic sets of $\varphi$ with $l=p / k$ such that all $\varphi \mid B_{i}: B_{i} \rightarrow B_{i}$ have period $k$, and the dynamical systems $\left(B_{i}, \varphi \mid B_{i}\right)$ are a factor of each other, and in particular they are conjugate if $\tau$ is a homeomorphism.

Then we prove an extension of a basic result in symbolic dynamics. Using this and many techniques in symbolic dynamics, we prove the following. If $\tau: X \rightarrow X$ is a topologically transitive, positively expansive onto continuous map having POTP, and $\varphi: X \rightarrow X$ is a positively expansive onto continuous map with $\varphi \tau=\tau \varphi$, then $\varphi$ has POTP. If $\tau: X \rightarrow X$ is a topologically transitive, expansive homeomorphism having POTP, and $\varphi: X \rightarrow X$ is a positively expansive onto continuous map with $\varphi \tau=\tau \varphi$, then $\varphi$ has POTP and is constant-to-one.

Further we define 'essentially LR endomorphisms' for systems of expansive onto continuous maps of compact metric spaces, and prove that if $\tau: X \rightarrow$ $X$ is an expansive homeomorphism with canonical coordinates and $\varphi$ is an essentially LR automorphism of $(X, \tau)$, then $\varphi$ has canonical coordinates. We add some discussions on basic properties of the essentially LR endomorphisms.
\end{abstract}

\section{INTRODUCTION}

We are interested in the interrelationships between the dynamical properties of commuting continuous maps of a compact metric space. Throughout this introduction, we assume that $\tau: X \rightarrow X$ and $\varphi: X \rightarrow X$ are onto continuous maps of a compact metric space with

$$
\tau \varphi=\varphi \tau .
$$

If $\tau$ is expansive, then $\varphi$ is an endomorphism of the expansive system $(X, \tau)$. Under what conditions and to what extent is a certain dynamical property of $\tau$ handed down to $\varphi$ ? Problems of this type arose in symbolic dynamics as problems on endomorphisms and automorphisms of subshifts or one-sided subshifts, that is, for

Received by the editors March 31, 1997 and, in revised form, November 13, 1998.

2000 Mathematics Subject Classification. Primary 54H20; Secondary 37B10, 37B15. 
the case where $X$ is 0-dimensional and $\tau$ is an expansive homeomorphism or a positively expansive continuous map. Boyle and Krieger $[\mathrm{BK}$ seem to be the first in the literature to consider such a problem; they proved that if $(X, \tau)$ and $(X, \varphi)$ are topological Markov shifts and if $\tau$ is topologically transitive (i.e., has a dense forward orbit) with period $p$, then for some $k$ dividing $p, \varphi$ is the union of $p / k$ disjoint conjugate topologically transitive topological Markov shifts of period $k$. The following questions were asked for the case that $X$ is 0 -dimensional, in terms of symbolic dynamics in Questions 2a, 3a(1), and 3c of [N1]. (a) If $\tau$ and $\varphi$ are expansive homeomorphisms and if $\tau$ has the pseudo-orbit tracing property (POTP), then does $\varphi$ have POTP? (b) If $\tau$ is an expansive homeomorphism having POTP and $\varphi$ is positively expansive, then does $\varphi$ have POTP? (c) If $\tau$ and $\varphi$ are positively expansive and $\tau$ has POTP, then does $\varphi$ have POTP? In [BL, Boyle and Lind introduced a symbolic dynamics method, an analogue of the block maps between subshifts, to systems of expansive homeomorphisms of compact metric spaces, and extensively studied the dynamics of commuting homeomorphisms. In particular, they showed that if $\tau$ and $\varphi$ are expansive homeomorphisms corresponding to two directions in the same 'expansive component' of directions for a $\mathbf{Z}^{d}$ action, then $\tau$ has POTP if and only if $\varphi$ does. Actually they more generally showed a similar fact for directions including irrational ones for $\mathbf{Z}^{d}$ actions on compact metric spaces ([BL], Proposition 8.3) and posed the question (a) ([BL], Problem 9.6).

In this paper, though we do not treat irrational directions, we also investigate similar phenomena in a different setting. But our main focus is rather on the questions (b) and (c). Recently in [N2] and independently in $[\mathrm{K}]$ (see $[\mathrm{BM}]$ and $[\mathrm{BFF}]$ ), the following have been proved under the assumption that $X$ is 0 -dimensional.

(1) If $\tau$ is a topologically transitive, expansive homeomorphism with POTP and $\varphi$ is positively expansive, then $\varphi$ has POTP.

(2) If $\tau$ and $\varphi$ are positively expansive with $\tau$ topologically transitive and if $\tau$ has POTP, then $\varphi$ has POTP.

We show that (1) and (2) can be proved without the assumption that $X$ is 0 dimensional (Theorems 3.5 and 3.4), though little seems to be known about the question of where $\tau$ and $\varphi$ satisfying the expansiveness part of the hypotheses of (1) or (2) exist when $X$ is of dimension greater than 0 and not a closed topological manifold. We should note that any positively expansive onto continuous map of any closed topological manifold has POTP (see [Hi]). Our proof is an extension of the proof of (1) and (2) given for the case that $X$ is 0 -dimensional in [N2]. We use the symbolic dynamics method of Boyle and Lind mentioned above and many techniques in symbolic dynamics, in particular, 'textile-orbit-systems', which have been introduced in [N2] as an analogue of textile systems introduced in [N1].

In (2) the condition that $\tau$ is topologically transitive, cannot generally be deleted, which has been shown in $[\mathrm{BFF}]$ by an example with $X$ 0-dimensional. Doris Fiebig $[F]$ has shown that in (1) the condition that $\tau$ is topologically transitive can be deleted when $X$ is 0-dimensional. As for the question (a), she has given an example with $X 0$-dimensional which shows that the answer for (a) is negative when $\tau$ is not topologically transitive $[\mathrm{F}$. Hence (a) is open now for the case that $\tau$ is topologically transitive. The following problem is also left unsolved even for the 0-dimensinal case (see [N1] and [BoMa]). (d) If $\tau$ is positively expansive and has POTP and if $\varphi$ is an expansive homeomorphism, then does $\varphi$ have POTP?

In Section 1, we give preliminaries, show that a simple application of the proof of the topological decomposition theorem (an extension of the spectral decomposition 
theorem due to Smale $[\mathrm{S}$ and Bowen [Bow3]) given by Aoki and Hiraide $\mathrm{AH}$, can prove that when $\tau$ is expansive and has POTP, if $\varphi$ is topologically mixing, then so is $\tau$ (Theorem 1.6), and show that a combination of it and the proof of Boyle and Krieger $[\mathrm{BK}]$ for their result mentioned above can prove the following satisfactory extension of this result. If $\tau$ and $\varphi$ are expansive and have POTP and if $\tau$ is topologically transitive with period $p$, then for some $k$ dividing $p,(X, \varphi)$ is the disjoint union of $p / k$ dynamical systems of topologically transitive onto continuous maps with POTP and period $k$ which are a factor of each other, and in particular which are conjugate if $\tau$ is a homeomorphism (Theorem 1.7).

In Section 2, we prove a key result (Theorem 2.2) for our proofs of (1) and (2), which is an extension of the well-known result in symbolic dynamics that any right [left] resolving graph-homomorphism between irreducible graphs with the same spectral radius is right [left] covering (cf. Proposition 8.2.2 of [LM]).

In Section 3, we prove (1) and (2) and obtain immediate consequences of them and the results of Section 1 (Theorems 3.8, 3.9, and 3.6).

In Section 4, using textile-orbit-systems we define 'essentially LR endomorphisms' for systems of expansive onto continuous maps of compact metric spaces. We prove that if $\tau$ is an expansive homeomorphism with canonical coordinates and $\varphi$ is an essentially LR, but not necessarily expansive, automorphism of $(X, \tau)$, then $\varphi$ has canonical coordinates (Theorem 4.3). This result is closely related with that of Boyle and Lind mentioned above. We add some discussions on basic properties of essentially LR endomorphisms of systems of expansive onto continuous maps of compact metric spaces; in particular, we give a block-map-like characterization of the essentially LR automorphisms (Proposition 4.4). We also prove that if $X$ is 0 dimensional and $\tau$ is an expansive homeomorphism, then $\varphi \tau^{n}$ is an essentially LR endomorphism of $(X, \tau)$ for some $n \geq 0$ if and only if $\varphi$ is right closing (Proposition 4.10).

I would like to thank the referee for his or her superb job. He quite carefully read the original manuscript and gave very valuable suggestions and comments together with correcting errors. As one of them, he pointed out that a short proof for Theorem 2.2 is possible. By his suggestions and ideas, the proof of Theorem 2.2 has been greatly shortened. I am also grateful to K. Hiraide and M. Shishikura for helpful conversations about dynamical systems on spaces of dimension greater than 0 .

The original version of this paper was written when I was with the Faculty of Engineering, Mie University. I express my thanks to Mrs. Miyoko Kawamura for the word processing of the manuscript. The revised version was prepared at Hiroshima University. I am grateful to S. Okui (at Mie University), M. Ito, and especially Y. Nishino for their excellent help which enabled my own word processing for the revision.

\section{ENDOMORPHISMS OF EXPANSIVE SYSTEMS}

We give preliminaries and two results on commuting continuous maps, one concerning the topological mixing property and the other concerning topological transitivity.

Standing convention: Throughout this paper, all the spaces we consider are compact metric spaces. If $X$ is a space, then it is assumed to be endowed with metric $d_{X}$ with which it is compact. We also assume that 'map' means 'continuous 
map'. We let $\boldsymbol{x}$ denote the bisequence $\left(x_{j}\right)_{j \in \mathbf{Z}}$ of points $x_{j}$ of a space. That is, $\boldsymbol{x}=\left(x_{j}\right)_{j \in \mathbf{Z}}$. Similarly we let $\boldsymbol{x}^{\prime}, \hat{\boldsymbol{y}}, \overline{\boldsymbol{z}}^{(k)}$ and so on denote $\left(x_{j}^{\prime}\right)_{j \in \mathbf{Z}},\left(\hat{y}_{j}\right)_{j \in \mathbf{Z}},\left(\bar{z}_{j}^{(k)}\right)_{j \in \mathbf{Z}}$ and so on, each of which is a bisequence of points of some space.

Let $f: X \rightarrow X$ be a map. A bisequence $\boldsymbol{x}$ of points of $X$ is called an orbit of $f$ if $f\left(x_{j}\right)=x_{j+1}$ for all $j \in \mathbf{Z}$. For $\epsilon>0$, we say that $f$ is $\epsilon$-expansive or $\epsilon$ is an expansive constant for $f$ if for any orbits $\boldsymbol{x}$ and $\boldsymbol{x}^{\prime}$ of $f$, if $d_{X}\left(x_{j}, x_{j}^{\prime}\right) \leq \epsilon$ for all $j \in \mathbf{Z}$ then $\boldsymbol{x}=\boldsymbol{x}^{\prime}$. We say that $f$ is positively $\epsilon$-expansive if for any $x, x^{\prime} \in X$, if $d_{X}\left(f^{j}(x), f^{j}\left(x^{\prime}\right)\right) \leq \epsilon$ for all $j \geq 0$ then $x=x^{\prime}$. We say that $f$ is expansive if $f$ is $\epsilon$-expansive for some $\epsilon>0$, and that $f$ is positively expansive if $f$ is positively $\epsilon$-expansive for some $\epsilon>0$.

For $\delta>0$, a bisequence $\boldsymbol{y}$ of points of $X$ is called a $\delta$-pseudo-orbit of $f$ if $d_{X}\left(f\left(y_{j}\right), y_{j+1}\right) \leq \delta$ for all $j \in \mathbf{Z}$. For $\epsilon>0$, an orbit $\boldsymbol{x}$ of $f$ is said to $\epsilon$-trace $\boldsymbol{y}$ if $d_{X}\left(x_{j}, y_{j}\right) \leq \epsilon$ for all $j \in \mathbf{Z}$. We say that $f$ has the pseudo-orbit tracing property (POTP) if for any $\epsilon>0$, there is $\delta>0$ such that any $\delta$-pseudo-orbit of $f$ is $\epsilon$-traced by some orbit of $f$.

Let $\mathcal{O}_{f}$ be the metric space of all orbits of $f$ endowed with the metric $d_{\mathcal{O}_{f}}$ defined by

$$
d_{\mathcal{O}_{f}}(\boldsymbol{x}, \boldsymbol{y})=\sup \left\{2^{-|j|} d_{X}\left(x_{j}, y_{j}\right) \mid j \in \mathbf{Z}\right\}, \quad \boldsymbol{x}, \boldsymbol{y} \in \mathcal{O}_{f} .
$$

Then $\mathcal{O}_{f}$ is compact. We define $\sigma_{f}: \mathcal{O}_{f} \rightarrow \mathcal{O}_{f}$ by

$$
\sigma_{f}(\boldsymbol{x})=\left(f\left(x_{j}\right)\right)_{j \in \mathbf{Z}}, \quad \boldsymbol{x} \in \mathcal{O}_{f} .
$$

Clearly $\sigma_{f}$ is a homeomorphism. Moreover, $h\left(\sigma_{f}\right)=h(f)$ ([Bow1, Proposition $5.2)$, where $h$ denotes topological entropy.

Let $\epsilon>0$. We define, for $\boldsymbol{x} \in \mathcal{O}_{f}$,

$$
V_{\epsilon}^{-}(\boldsymbol{x}, f)=\left\{\boldsymbol{y} \in \mathcal{O}_{f} \mid d_{X}\left(x_{j}, y_{j}\right) \leq \epsilon \text { for all } j \leq 0\right\}
$$

and

$$
V_{\epsilon}^{+}(\boldsymbol{x}, f)=\left\{\boldsymbol{y} \in \mathcal{O}_{f} \mid d_{X}\left(x_{j}, y_{j}\right) \leq \epsilon \text { for all } j \geq 0\right\} .
$$

We say that $f$ has canonical coordinates (CC) if for any $\epsilon>0$, there is $\delta>0$ such that for any $\boldsymbol{x}$ and $\boldsymbol{y}$ in $\mathcal{O}_{f}$,

$$
d_{X}\left(x_{0}, y_{0}\right) \leq \delta \Rightarrow V_{\epsilon}^{-}(\boldsymbol{x}, f) \cap V_{\epsilon}^{+}(\boldsymbol{y}, f) \neq \emptyset .
$$

It is clear that if $f$ has POTP, then $f$ has CC. It is well known that if $f$ is expansive and has CC, then $f$ has POTP (see, e.g., [AS] or [Hi]).

In line with the definition of 'coding' given by Boyle and Lind [BL], we define the following. Let $A=\left\{\phi_{i}: X \rightarrow X_{i} \mid i \in I\right\}$ and $B=\left\{\phi_{j}^{\prime}: X \rightarrow X_{j}^{\prime} \mid j \in J\right\}$ be two sets of maps, where $I$ and $J$ are indexing sets. Let $\epsilon, \epsilon^{\prime}>0$. We say that $A\left(\epsilon, \epsilon^{\prime}\right)$-codes $B$ if for any $x, y \in X$,

$$
\forall i \in I, d_{X_{i}}\left(\phi_{i}(x), \phi_{i}(y)\right) \leq \epsilon \Rightarrow \forall j \in J, d_{X_{j}^{\prime}}\left(\phi_{j}^{\prime}(x), \phi_{j}^{\prime}(y)\right) \leq \epsilon^{\prime} .
$$

If $A(\epsilon, \epsilon)$-codes $B$, then we say that $A \epsilon$-codes $B$. (If $A$ or $B$ is a singleton set of a map, then we will abuse the map to denote the set.)

The following lemma is well known or a trivial extension of a well-known fact, but it is a key observation for the symbolic dynamics method introduced by Boyle and Lind $[\mathrm{BL}]$ for studying endomorphisms and automorphisms of expansive systems on compact metric spaces. 
Lemma 1.1. Let $\epsilon>0$. Let $f: X \rightarrow X$ and $\phi: X \rightarrow Y$ be onto maps.

(1) If $f$ is $\epsilon$-expansive, then for any $\epsilon^{\prime}>0$ there is an integer $m \geq 0$ such that $\left\{f^{j} \mid 0 \leq j \leq 2 m\right\}\left(\epsilon, \epsilon^{\prime}\right)$-codes $\phi f^{m}$ (i.e., for any $x, x^{\prime} \in X$, if $d_{X}\left(f^{j}(x), f^{j}\left(x^{\prime}\right)\right) \leq \epsilon$ for $0 \leq j \leq 2 m$, then $\left.d_{Y}\left(\phi f^{m}(x), \phi f^{m}\left(x^{\prime}\right)\right) \leq \epsilon^{\prime}\right)$.

(2) If $f$ is positively $\epsilon$-expansive, then, for any $\epsilon^{\prime}>0$, there is an integer $m \geq 0$ such that $\left\{f^{j} \mid 0 \leq j \leq m\right\}\left(\epsilon, \epsilon^{\prime}\right)$-codes $\phi$.

Let $f: X \rightarrow X$ and $g: Y \rightarrow Y$ be onto maps. Let $\epsilon>0$. Let $m, n$ be nonnegative integers. Then a factor map $\phi:(X, f) \rightarrow(Y, g)$ between dynamical systems is said to be of $(m, n)$ type with respect to $\epsilon$ if $\left\{f^{j} \mid j=0, \ldots, m+n\right\} \epsilon$-codes $g^{m} \phi$.

Throughout this paper we do not assume that a factor map is necessarily onto.

Boyle and Lind gave a 'finite' version of expansiveness which is an analogue of the block maps of symbolic dynamics ([BL], Lemma 3.2). The following is a version of it and a direct corollary to Lemma 1.1.

Corollary 1.2 (Boyle and Lind). Let $f: X \rightarrow X$ and $g: Y \rightarrow Y$ be onto maps. Let $\phi:(X, f) \rightarrow(Y, g)$ be a factor map. Let $\epsilon>0$.

(1) If $f$ is $\epsilon$-expansive, then there are integers $m, n \geq 0$ such that $\phi$ is of $(m, n)$ type with respect to $\epsilon$.

(2) If $f$ is positively $\epsilon$-expansive, then there is an integer $n \geq 0$ such that $\phi$ is of $(0, n)$ type with respect to $\epsilon$.

An onto map $f: X \rightarrow X$ is said to be (one-sided) topologically transitive if for any nonempty open sets $U, V \subset X$, there is $n \in \mathbf{N}$ such that $f^{n}(U) \cap V \neq \emptyset$.

By Bowen's theory (Bow2, Bow4, Bow5]) the following is known (see [AH]): if $f: X \rightarrow X$ is a topologically transitive, expansive homeomorphism with POTP, then there exist an irreducible topological Markov shift $\sigma: \Sigma \rightarrow \Sigma$ and a boundedto-one factor map of $(\Sigma, \sigma)$ onto $(X, f)$, and hence $(X, f)$ is a finitely presented system, in the sense of Fried [Fr], with canonical coordinates. It is known (see Section 1 of [Boy]) that if $f: X \rightarrow X$ and $g: Y \rightarrow Y$ are topologically transitive, expansive homeomorphisms and have POTP and if $\phi:(X, f) \rightarrow(Y, g)$ is an onto factor map, then $h(f)=h(g)$ if and only if $\phi$ is bounded-to-one. This is considered to be a natural extension of a well-known property of the factor maps between irreducible topological Markov shifts (cf. [CP]).

Lemma 1.3. Let $f: X \rightarrow X$ and $g: Y \rightarrow Y$ be onto maps. Let $\phi:(X, f) \rightarrow(Y, g)$ be an onto factor map. Let $\tilde{\phi}:\left(\mathcal{O}_{f}, \sigma_{f}\right) \rightarrow\left(\mathcal{O}_{g}, \sigma_{g}\right)$ be the factor map induced by $\phi$, i.e., for each $\boldsymbol{x}=\left(x_{j}\right)_{j \in \mathbf{Z}} \in \mathcal{O}_{f}, \tilde{\phi}(\boldsymbol{x})=\left(\phi\left(x_{j}\right)\right)_{j \in \mathbf{Z}}$. Then $\tilde{\phi}$ is onto.

Proof. Let $\boldsymbol{y} \in \mathcal{O}_{g}$. Since $\phi$ is onto, for each $j \in \mathbf{Z}$ there is $x_{j} \in X$ with $\phi\left(x_{j}\right)=y_{j}$. Since $f$ is onto, for each $j \in \mathbf{Z}$ there is $\boldsymbol{x}^{(j)} \in \mathcal{O}_{f}$ such that $x_{j}^{(j)}=x_{j}$ and hence $x_{j+l}^{(j)}=f^{l}\left(x_{j}\right)$ for all $l \geq 0$. Since $\mathcal{O}_{f}$ is compact, there are a sequence $0>j_{1}>$ $j_{2}>\cdots$ of integers and a point $\overline{\boldsymbol{x}}$ of $\mathcal{O}_{f}$ such that $\lim _{i \rightarrow \infty} \boldsymbol{x}^{\left(j_{i}\right)}=\overline{\boldsymbol{x}}$. We shall show that $\tilde{\phi}(\overline{\boldsymbol{x}})=\boldsymbol{y}$.

Let $\epsilon>0$. Let $\delta>0$ be such that $\operatorname{id}_{X}(\delta, \epsilon)$-codes $\phi$. Let $r \in \mathbf{Z}$. There is $k \in \mathbf{N}$ such that $j_{k}<r$ and $d_{\mathcal{O}_{f}}\left(\boldsymbol{x}^{\left(j_{k}\right)}, \overline{\boldsymbol{x}}\right) \leq 2^{-|r|} \delta$. Since $\sup \left\{2^{-|i|} d_{X}\left(x_{i}^{\left(j_{k}\right)}, \bar{x}_{i}\right) \mid i \in \mathbf{Z}\right\} \leq$ $2^{-|r|} \delta$, we have $d_{X}\left(x_{r}^{\left(j_{k}\right)}, \bar{x}_{r}\right) \leq \delta$, so that $d_{Y}\left(\phi\left(x_{r}^{\left(j_{k}\right)}\right), \phi\left(\bar{x}_{r}\right)\right) \leq \epsilon$. Since $j_{k}<r$, we have $\phi\left(x_{r}^{\left(j_{k}\right)}\right)=\phi\left(f^{r-j_{k}}\left(x_{j_{k}}\right)\right)=g^{r-j_{k}}\left(y_{j_{k}}\right)=y_{r}$. Thus $d_{Y}\left(y_{r}, \phi\left(\bar{x}_{r}\right)\right) \leq \epsilon$. Since $\epsilon>0$ and $r \in \mathbf{Z}$ are arbitrary, we have $\boldsymbol{y}=\tilde{\phi}(\overline{\boldsymbol{x}})$. 
Lemma 1.4. Let $f: X \rightarrow X$ and $g: Y \rightarrow Y$ be topologically transitive, expansive, onto maps with $h(f)=h(g)$ and with both having POTP. If $\phi:(X, f) \rightarrow(Y, g)$ is an onto factor map, then $\tilde{\phi}$ is bounded-to-one.

Proof. The hypotheses imply that $\sigma_{f}: \mathcal{O}_{f} \rightarrow \mathcal{O}_{f}$ and $\sigma_{g}: \mathcal{O}_{g} \rightarrow \mathcal{O}_{g}$ are expansive, topologically transitive homeomorphisms and have POTP (see [AH]). Hence $h\left(\sigma_{f}\right)=h(f)=h(g)=h\left(\sigma_{g}\right)$. Since $\phi$ is onto, $\tilde{\phi}:\left(\mathcal{O}_{f}, \sigma_{f}\right) \rightarrow\left(\mathcal{O}_{g}, \sigma_{g}\right)$ is onto, by Lemma 1.3. Hence by the fact stated before Lemma 1.3, $\tilde{\phi}$ is bounded-to-one.

Let $f: X \rightarrow X$ be a map. We say that $x \in X$ is a nonwandering point of $f$ if for any open neighborhood $U$ of $x$ there is $n \in \mathbf{N}$ such that $f^{n}(U) \cap U \neq \emptyset$. Let $\Omega(f)$ denote the set of all nonwandering points of $f$. We say that $f$ is topologically mixing if for any nonempty open sets $U, V \subset X$ there is $N \in \mathbf{N}$ such that $U \cap f^{n}(V) \neq \emptyset$ for all $n \geq N$.

The following 'topological decomposition theorem' was given by Aoki and Hiraide AH] as an extension of the 'spectral decomposition theorem' due to Smale [S] and Bowen Bow3.

Theorem 1.5 (Aoki and Hiraide $[\mathrm{AH}]$ ). Let $f: X \rightarrow X$ be an expansive onto map with POTP.

(1) $\Omega(f)$ contains finitely many closed subsets $B_{i}, 1 \leq i \leq l$, such that (i) $B_{i} \cap$ $B_{j}=\emptyset$ if $i \neq j$, and $f\left(B_{i}\right)=B_{i}$, (ii) $\Omega(f)=\bigcup_{i=1}^{l} B_{i}$, and (iii) $f \mid B_{i}: B_{i} \rightarrow B_{i}$ $i$ topologically transitive and has POTP (the subsets $B_{i}$ are called the basic sets of f).

(2) For a basic set $B$ of $f$, there exist $a \in \mathbf{N}$ and closed subsets $C_{i}, 0 \leq i \leq a-1$, such that ( $i) C_{i} \cap C_{j}=\emptyset$ if $i \neq j$, and $f\left(C_{i}\right)=C_{i+1 \bmod a}$, (ii) $B=\bigcup_{i=0}^{a-1} C_{i}$, and (iii) $f^{a} \mid C_{i}: C_{i} \rightarrow C_{i}$ is topologically mixing and has POTP (the subsets $C_{i}$ are called the elementary sets in $B$ ).

Theorem 1.6. Let $f: X \rightarrow X$ be an expansive onto map with POTP. If there is a topologically mixing map $g: X \rightarrow X$ with $f g=g f$, then $f$ is topologically mixing.

Proof. For $\delta>0$ and $x, y \in X$, we write $x \stackrel{\delta}{\sim} y$ to mean that there are sequences $x_{0}, \ldots, x_{k}$ and $y_{0}, \ldots, y_{l}$ of points of $X$ with $k, l \geq 0$ such that

$$
\begin{aligned}
& x_{0}=x, \quad d_{X}\left(f\left(x_{i}\right), x_{i+1}\right)<\delta \text { for } 0 \leq i \leq k-1, \quad x_{k}=y, \\
& y_{0}=y, \quad d_{X}\left(f\left(y_{i}\right), y_{i+1}\right)<\delta \quad \text { for } 0 \leq i \leq l-1, \quad y_{l}=x .
\end{aligned}
$$

We write $x \sim y$ to mean that $x \stackrel{\delta}{\sim} y$ for any $\delta>0$. It is found in Section 3.4 of [AH] that $\sim$ is an equivalence relation on $\Omega(f)$ and the equivalence classes constitute the basic sets of $f$. Since $f g=g f$ and $g$ is uniformly continuous, it follows that for $x, y \in \Omega(f)$, if $x \sim y$, then $g(x) \sim g(y)$.

Let $\operatorname{Per}(f)$ denote the set of all periodic points of $f$. For $p \in \operatorname{Per}(f)$, let $B(p)$ be the basic set of $f$ containing $p$, and let $C_{p}$ be the subset $\operatorname{cl}\left(W^{s}(p, f) \cap B(p)\right)$ with

$$
W^{s}(p, f)=\left\{x \in X \mid \lim _{n \rightarrow \infty} d_{X}\left(f^{n}(p), f^{n}(x)\right)=0\right\} .
$$

As is found in Section 3.4 of $[\mathrm{AH}], C_{p}$ is an elementary set in $B(p)$ for $p \in \operatorname{Per}(f)$. Since $f g=g f$, we have $g(p) \in \operatorname{Per}(f)$ for $p \in \operatorname{Per}(f)$.

Let $p \in \operatorname{Per}(f)$. Then by the above, if $x \in B(p)$, then $g(x) \in B(g(p))$. If $x \in W^{s}(p, f)$, then $g(x) \in W^{s}(g(p), f)$. Therefore

$$
g\left(W^{s}(p, f) \cap B(p)\right) \subset W^{s}(g(p), f) \cap B(g(p)) .
$$


Thus

$$
\begin{aligned}
g\left(C_{p}\right)=g\left(\operatorname{cl}\left(W^{s}(p, f) \cap B(p)\right)\right) & \subset \operatorname{cl}\left(g\left(W^{s}(p, f) \cap B(p)\right)\right) \\
& \subset \operatorname{cl}\left(W^{s}(g(p), f) \cap B(g(p))\right)=C_{g(p)} .
\end{aligned}
$$

Since there are only finitely many elementary sets in the basic sets of $f$, there are integers $0 \leq i<j$ such that $C_{g^{i}(p)}=C_{g^{j}(p)}$. Hence

$$
g^{j-i}\left(C_{g^{i}(p)}\right) \subset C_{g^{j}(p)}=C_{g^{i}(p)} .
$$

Thus if $C_{g^{i}(p)} \neq X$, then $g^{j-i}$ would not be topologically transitive. But this is not the case, because $g$ is topologically mixing. Therefore we have $C_{g^{i}(p)}=X$, which implies that $f$ is topologically mixing.

If $f: X \rightarrow X$ is a topologically transitive, expansive onto map with POTP, then $X$ itself is a basic set of $f$. We call the number of elementary sets in the basic set $X$ the period of $f$.

The following theorem is a generalization of a result of Boyle and Krieger (Lemma 2.13 of $[\mathrm{BK}])$.

Theorem 1.7. Let $f: X \rightarrow X$ and $g: X \rightarrow X$ be commuting expansive onto maps with POTP. Suppose that $f$ is topologically transitive with period $r$. Then, for some $k$ dividing $r, X=\bigcup_{i=0}^{l-1} B_{i}$, where $B_{i}, 0 \leq i \leq l-1$, are the basic sets of $g$ with $l=r / k$ such that all $g \mid B_{i}: B_{i} \rightarrow B_{i}$ have period $k$, and the dynamical systems $\left(B_{i}, g \mid B_{i}\right)$ are a factor of each other, and in particular they are conjugate to each other if $f$ is a homeomorphism.

Proof. The proof is a natural extension of the proof of Lemma 2.13 of [BK]. We use the same notation as in the proof of Theorem 1.6.

Let $p \in \operatorname{Per}(f)$. As is found in Section 3.4 of $[\mathrm{AH}], C_{p}, C_{f(p)}, \ldots, C_{f^{r-1}(p)}$ are the elementary sets of $f$ with $C_{f^{r}(p)}=C_{p}$. There is $0 \leq L \leq r-1$ with $g(p) \in$ $C_{f^{L}(p)}$. Since $g\left(f^{i}(p)\right) \in f^{i}\left(C_{f^{L}(p)}\right)=C_{f^{i+L}(p)}$ and $g f^{i}(p) \in \operatorname{Per}(f)$, it follows that $C_{g f^{i}(p)}=C_{f^{i+L}(p)}$ for $0 \leq i \leq r-1$. By the proof of Theorem 1.6, for $0 \leq i \leq r-1$, we have $C_{g f^{i}(p)} \supset g\left(C_{f^{i}(p)}\right)$, so that $C_{g f^{i}(p)}=g\left(C_{f^{i}(p)}\right)$ because $g$ is onto. Thus we have

$$
g\left(C_{f^{i}(p)}\right)=C_{f^{i+L}(p)} \quad \text { for } 0 \leq i \leq r-1 .
$$

Since $f$ and $g$ have POTP and $C_{f^{i}(p)}, 0 \leq i \leq r-1$, are disjoint closed sets each of which is $f^{r}$-invariant and $g^{r}$-invariant, it follows that $f^{r} \mid C_{f^{i}(p)}$ and $g^{r} \mid C_{f^{i}(p)}$ have POTP for $0 \leq i \leq r-1$. By Theorem 1.5, $f^{r} \mid C_{f^{i}(p)}$ is topologically mixing for $0 \leq i \leq r-1$. Therefore by Theorem $1.6, g^{r} \mid C_{f^{i}(p)}$ is topologically mixing for $0 \leq i \leq r-1$.

Let $l=\operatorname{gcd}(r, L)$ and let $k=r / l$. Then for each $i$, the sets

$$
g^{j}\left(C_{f^{i}(p)}\right)=C_{f^{i+j L}(p)}, \quad 0 \leq j \leq k-1,
$$

are disjoint closed sets and $g^{k}\left(C_{f^{i}(p)}\right)=C_{f^{i}(p)}$. Since $g^{k} \mid C_{f^{i}(p)}$ has POTP and $g^{r} \mid C_{f^{i}(p)}$ is topologically mixing, it follows from Theorem 1.6 that $g^{k} \mid C_{f^{i}(p)}$ is topologically mixing. Let

$$
B_{i}=\bigcup_{j=0}^{k-1} g^{j}\left(C_{f^{i}(p)}\right), \quad 0 \leq i \leq l-1 .
$$


Then $\bigcup_{i=0}^{l-1} B_{i}=X$, with the sets $B_{i}$ disjoint, closed, and $g$-invariant, and each $g \mid B_{i}$ topologically transitive. Therefore $B_{i}, 0 \leq i \leq l-1$, are the basic sets of $g$, and $g^{j}\left(C_{f^{i}(p)}\right), 0 \leq j \leq k-1$, are the elementary sets in $B_{i}$, so that $g \mid B_{i}$ has period $k$ for $0 \leq i \leq l-1$.

For any $0 \leq i, j \leq l-1$, since $f^{j-i+L}\left(B_{i}\right)=B_{j}$ and $g f^{j-i+L}=f^{j-i+L} g$, $\left(B_{j}, g \mid B_{j}\right)$ is a factor of $\left(B_{i}, g \mid B_{i}\right)$, and if in particular $f$ is a homeomorphism, then $\left(B_{j}, g \mid B_{j}\right)$ is conjugate to $\left(B_{i}, g \mid B_{i}\right)$.

\section{ReSOLVING FACTOR MAPS}

Let $f: X \rightarrow X$ and $g: Y \rightarrow Y$ be onto maps. Let $\epsilon>0$. Let $\phi:(X, f) \rightarrow(Y, g)$ be a factor map. We say that $\phi$ is $\epsilon$-closeness preserving, or $\epsilon$-preserving for short, if $\operatorname{id}_{X} \epsilon$-codes $\phi$. The notion of an $\epsilon$-preserving factor map is an analogy of that of a one-block map in symbolic dynamics. It is easily proved by using Corollary 1.2 that if $f$ is expansive and $\epsilon$ is an expansive constant for $f$, then, as every block map between subshifts becomes a one-block map passing through a higher block system of sufficiently large order, $\phi$ becomes $\epsilon$-preserving passing through a higher block system $\left(X^{\prime}, f^{\prime}\right)$ of sufficiently large order of $(X, f)$ (see the second paragraph of the next section), that is, there is a natural conjugacy $\rho:(X, f) \rightarrow\left(X^{\prime}, f^{\prime}\right)$ such that $\phi \rho^{-1}$ is an $\epsilon$-preserving factor map of $\left(X^{\prime}, f^{\prime}\right)$ into $(Y, g)$.

Let $\phi$ be $\epsilon$-preserving. We say that $\phi$ is right $\epsilon$-resolving if $\left\{\operatorname{id}_{X}, g \phi\right\} \epsilon$-codes $f$, and $\phi$ is said to be left $\epsilon$-resolving if $\{\phi, f\} \epsilon$-codes $\operatorname{id}_{X}$.

Let $\tilde{\phi}:\left(\mathcal{O}_{f}, \sigma_{f}\right) \rightarrow\left(\mathcal{O}_{g}, \sigma_{g}\right)$ be the factor map induced by $\phi$, i.e., for $\boldsymbol{x} \in$ $\mathcal{O}_{f}, \tilde{\phi}(\boldsymbol{x})=\left(\phi\left(x_{j}\right)\right)_{j \in \mathbf{Z}}$. Since $\phi$ is $\epsilon$-preserving, for every $\boldsymbol{x} \in \mathcal{O}_{f}$,

$$
V_{\epsilon}^{-}(\tilde{\phi}(\boldsymbol{x}), g) \supset \tilde{\phi}\left(V_{\epsilon}^{-}(\boldsymbol{x}, f)\right) \text { and } V_{\epsilon}^{+}(\tilde{\phi}(\boldsymbol{x}), g) \supset \tilde{\phi}\left(V_{\epsilon}^{+}(\boldsymbol{x}, f)\right) \text {. }
$$

We say that $\phi$ is right $\epsilon$-complete, if for all $\boldsymbol{x} \in \mathcal{O}_{f}$,

$$
V_{\epsilon}^{-}(\tilde{\phi}(\boldsymbol{x}), g)=\tilde{\phi}\left(V_{\epsilon}^{-}(\boldsymbol{x}, f)\right) \text {. }
$$

We also say that $\phi$ is left $\epsilon$-complete, if for all $\boldsymbol{x} \in \mathcal{O}_{f}$,

$$
V_{\epsilon}^{+}(\tilde{\phi}(\boldsymbol{x}), g)=\tilde{\phi}\left(V_{\epsilon}^{+}(\boldsymbol{x}, f)\right) \text {. }
$$

For $\boldsymbol{x} \in \mathcal{O}_{f}$, let $\tilde{\phi}_{\epsilon, \boldsymbol{x}}^{-}: V_{\epsilon}^{-}(\boldsymbol{x}, f) \rightarrow V_{\epsilon}^{-}(\tilde{\phi}(\boldsymbol{x}), g)$ be defined as the restriction of $\tilde{\phi}$, and let $\tilde{\phi}_{\epsilon, \boldsymbol{x}}^{+}: V_{\epsilon}^{+}(\boldsymbol{x}, f) \rightarrow V_{\epsilon}^{+}(\tilde{\phi}(\boldsymbol{x}), g)$ be similarly defined. Then we say that $\phi$ is right $\epsilon$-closing if $\tilde{\phi}_{\epsilon, \boldsymbol{x}}^{-}$is 1-1 for all $\boldsymbol{x} \in \mathcal{O}_{f}$, and $\phi$ is said to be left $\epsilon$-closing if $\tilde{\phi}_{\epsilon, \boldsymbol{x}}^{+}$ is 1-1 for all $\boldsymbol{x} \in \mathcal{O}_{f}$. Hence we see that $\phi$ is right $\epsilon$-closing and right $\epsilon$-complete if and only if $\tilde{\phi}_{\epsilon, \boldsymbol{x}}^{-}$is bijective for all $\boldsymbol{x} \in \mathcal{O}_{f}$, and the left version of this also holds. As is easily seen by using Lemma 1.1, when $f$ is $\epsilon$-expansive, if $\phi$ is right $\epsilon$-resolving, then it is right $\epsilon$-closing, and if $\phi$ is left $\epsilon$-resolving, then it is left $\epsilon$-closing.

Proposition 2.1. Let $f: X \rightarrow X$ and $g: Y \rightarrow Y$ be onto maps. Let $\epsilon>0$. Let $g$ be $\epsilon$-expansive. Let $\phi:(X, f) \rightarrow(Y, g)$ be an $\epsilon$-preserving conjugacy.

(1) If $\phi$ is right $\epsilon$-resolving, then it is right $\epsilon$-complete.

(2) If $\phi$ is left $\epsilon$-resolving, then it is left $\epsilon$-complete.

Proof. To prove (1), let $\boldsymbol{x}, \boldsymbol{x}^{\prime} \in \mathcal{O}_{f}$ with $\tilde{\phi}\left(\boldsymbol{x}^{\prime}\right) \in V_{\epsilon}^{-}(\tilde{\phi}(\boldsymbol{x}), g)$. We shall show that $\boldsymbol{x}^{\prime} \in V_{\epsilon}^{-}(\boldsymbol{x}, f)$. Since $\mathcal{O}_{f}$ is compact, there are a sequence $0<k_{1}<k_{2}<\cdots$ of integers and points $\overline{\boldsymbol{x}}, \overline{\boldsymbol{x}}^{\prime}$ of $\mathcal{O}_{f}$ such that $\lim _{i \rightarrow \infty} \sigma_{f}^{-k_{i}}(\boldsymbol{x})=\overline{\boldsymbol{x}}$ and $\lim _{i \rightarrow \infty} \sigma_{f}^{-k_{i}}\left(\boldsymbol{x}^{\prime}\right)=$ $\overline{\boldsymbol{x}}^{\prime}$. Let $\overline{\boldsymbol{y}}=\tilde{\phi}(\overline{\boldsymbol{x}})$ and let $\overline{\boldsymbol{y}}^{\prime}=\tilde{\phi}\left(\overline{\boldsymbol{x}}^{\prime}\right)$. Let $r \in \mathbf{Z}$. Then 


$$
\sigma_{g}^{r}(\overline{\boldsymbol{y}})=\sigma_{g}^{r}\left(\tilde{\phi}\left(\lim _{i \rightarrow \infty} \sigma_{f}^{-k_{i}}(\boldsymbol{x})\right)\right)=\lim _{i \rightarrow \infty} \sigma_{g}^{r-k_{i}} \tilde{\phi}(\boldsymbol{x}) \quad \text { and } \quad \sigma_{g}^{r}\left(\overline{\boldsymbol{y}}^{\prime}\right)=\lim _{i \rightarrow \infty} \sigma_{g}^{r-k_{i}} \tilde{\phi}\left(\boldsymbol{x}^{\prime}\right) \text {. }
$$

Since $\tilde{\phi}\left(\boldsymbol{x}^{\prime}\right) \in V_{\epsilon}^{-}(\tilde{\phi}(\boldsymbol{x}), g)$, for all $i$ with $k_{i} \geq r$, the distance between the 0 -th coordinate of $\sigma_{g}^{r-k_{i}} \tilde{\phi}(\boldsymbol{x})$ and that of $\sigma_{g}^{r-k_{i}} \tilde{\phi}\left(\boldsymbol{x}^{\prime}\right)$ is not greater than $\epsilon$. Hence so is the distance between the 0-th coordinate of $\sigma_{g}^{r}(\overline{\boldsymbol{y}})$ and that of $\sigma_{g}^{r}\left(\overline{\boldsymbol{y}}^{\prime}\right)$, i.e., $d_{Y}\left(\bar{y}_{r}, \bar{y}_{r}^{\prime}\right) \leq$ $\epsilon$. Since $r \in \mathbf{Z}$ is arbitrary and $g$ is $\epsilon$-expansive, we have $\overline{\boldsymbol{y}}=\overline{\boldsymbol{y}}^{\prime}$, so that $\overline{\boldsymbol{x}}=\overline{\boldsymbol{x}}^{\prime}$ because $\tilde{\phi}$ is 1-1. Thus $d_{\mathcal{O}_{f}}\left(\sigma_{f}^{-k_{i}}(\boldsymbol{x}), \sigma_{f}^{-k_{i}}\left(\boldsymbol{x}^{\prime}\right)\right) \leq \epsilon$ for all sufficiently large $i$, so that $d_{X}\left(x_{-k_{i}}, x_{-k_{i}}^{\prime}\right) \leq \epsilon$ for all sufficiently large $i$. Therefore, since $d_{Y}\left(\phi\left(x_{j}\right), \phi\left(x_{j}^{\prime}\right)\right) \leq \epsilon$ for all $j \leq 0$ and $\phi$ is right $\epsilon$-resolving, it follows that $d_{X}\left(x_{j}, x_{j}^{\prime}\right) \leq \epsilon$ for all $j \leq 0$, which implies that $\boldsymbol{x}^{\prime} \in V_{\epsilon}^{-}(\boldsymbol{x}, f)$.

The proof of (2) is similar to the above.

To prove the main theorem of this section, it is convenient to introduce some notations. Let $\delta, \epsilon>0$. Let $f: X \rightarrow X$ be a $2 \epsilon$-expansive, onto map such that any $\delta$-pseudo-orbit of $f$ is $\epsilon$-traced by some orbit of $f$. This orbit is unique for the $\delta$-pseudo-orbit because $f$ is $2 \epsilon$-expansive. For $\boldsymbol{x} \in \mathcal{O}_{f}$ and $k, l \in \mathbf{Z}$ with $k<l$, let $\boldsymbol{x}_{[k, l)}, \boldsymbol{x}_{(-\infty, l)}$, and $\boldsymbol{x}_{[k, \infty)}$ denote the finite, left infinite, and right infinite sequences $\left(x_{j}\right)_{k \leq j<l},\left(x_{j}\right)_{j<l}$, and $\left(x_{j}\right)_{j \geq k}$, respectively. Let $\boldsymbol{x}^{(0)}, \ldots, \boldsymbol{x}^{(n)} \in \mathcal{O}_{f}$ with $n \in \mathbf{N}$. Let $l_{0}, k_{1}, l_{1}, \ldots, k_{n-1}, l_{n-1}, k_{n} \in \mathbf{Z}$ with $k_{i}<l_{i}, i=1, \ldots, n-1$. Then an expression $E$ of the form

$$
E=\boldsymbol{x}_{\left(-\infty, l_{0}\right)^{*}}^{(0)} \stackrel{\delta}{\circ} \boldsymbol{x}_{\left[k_{1}, l_{1}\right)}^{(1)} \stackrel{\delta}{\circ} \ldots \stackrel{\delta}{\circ} \boldsymbol{x}_{\left[k_{n-1}, l_{n-1}\right)}^{(n-1)} \stackrel{\delta}{\circ} \boldsymbol{x}_{\left[k_{n}, \infty\right)}^{(n)}
$$

means that $d_{X}\left(x_{l_{i-1}}^{(i-1)}, x_{k_{i}}^{(i)}\right) \leq \delta$ for $i=1, \ldots, n$ and denotes the $\delta$-pseudo-orbit $\boldsymbol{y}$ of $f$ such that $y_{j}=x_{j}^{(0)}$ for $j<l_{0}$, and for $i=1, \ldots, n, y_{\left(\sum_{m=0}^{i-1} l_{m}\right)-\left(\sum_{m=1}^{i} k_{m}\right)+j}=$ $x_{j}^{(i)}$ for $k_{i} \leq j<l_{i}$, with $l_{n}=\infty$. Hence $*$ indicates the real time interval. Let trace $_{\epsilon}(E)$ denote the orbit of $f$ which $\epsilon$-traces the $\delta$-pseudo-orbit denoted by $E$. Let $\phi:(X, f) \rightarrow(Y, g)$ be an onto factor map between dynamical systems, where $f: X \rightarrow X$ and $g: Y \rightarrow Y$ are topologically transitive, expansive, onto maps with the same topological entropy and with $f$ having POTP. For convenience we define a constant-triple for $\phi$ to be the triple $\left(c, \epsilon_{0}, \delta_{0}\right)$ of positive numbers such that both $f$ and $g$ are $c$-expansive, $\operatorname{id}_{X}\left(\epsilon_{0}, c / 2\right)$-codes $\phi$ with $\epsilon_{0} \leq c$, and any $\delta_{0}$-pseudo-orbit of $f$ is $\epsilon_{0}$-traced by some orbit of $f$ with $\delta_{0} \leq \epsilon_{0}$.

Theorem 2.2. Let $f: X \rightarrow X$ and $g: Y \rightarrow Y$ be topologically transitive, expansive, onto maps with $h(f)=h(g)$ and both having POTP. Let $\phi:(X, f) \rightarrow(Y, g)$ be an onto factor map. If $\epsilon>0$ is sufficiently small, that is, if there is a constant-triple $\left(c, \epsilon_{0}, \delta_{0}\right)$ for $\phi$ with $\epsilon<\delta_{0} / 2$, then the following hold.

(1) If $\phi$ is $\epsilon$-preserving and right $\epsilon$-resolving, then $\phi$ is right $\epsilon$-complete.

(2) If $\phi$ is $\epsilon$-preserving and left $\epsilon$-resolving, then $\phi$ is left $\epsilon$-complete.

Proof. Let $\tilde{\phi}:\left(\mathcal{O}_{f}, \sigma_{f}\right) \rightarrow\left(\mathcal{O}_{g}, \sigma_{g}\right)$ be the factor map induced by $\phi$. By Lemmas 1.3 and 1.4, $\tilde{\phi}$ is bounded-to-one and onto. To prove (1), assume that $\phi$ is $\epsilon$-preserving and right $\epsilon$-resolving. Let $\boldsymbol{y}$ be a point of $\mathcal{O}_{g}$ with the maximum number of inverses, say $\boldsymbol{x}^{(1)}, \ldots, \boldsymbol{x}^{(n)}$, under $\tilde{\phi}$. We claim that

$$
\tilde{\phi}^{-1}\left(V_{\epsilon}^{-}(\boldsymbol{y}, g)\right)=\bigcup_{i=1}^{n} V_{\epsilon}^{-}\left(\boldsymbol{x}^{(i)}, f\right) .
$$


For otherwise there would be $\boldsymbol{x}^{\prime} \notin \bigcup_{i=1}^{n} V_{\epsilon}^{-}\left(\boldsymbol{x}^{(i)}, f\right)$ with $\tilde{\phi}\left(\boldsymbol{x}^{\prime}\right) \in V_{\epsilon}^{-}(\boldsymbol{y}, g)$. Since $\mathcal{O}_{f}$ is compact, there is a sequence $0<k_{1}<k_{2}<\ldots$ of integers such that the sequences $\left(\sigma_{f}-k_{j}\left(\boldsymbol{x}^{(i)}\right)\right)_{j \in \mathbf{N}}, i=1, \ldots, n$, and $\left(\sigma_{f}-k_{j}\left(\boldsymbol{x}^{\prime}\right)\right)_{j \in \mathbf{N}}$ converge. Let their limits be $\overline{\boldsymbol{x}}^{(i)}, i=1, \ldots, n$, and $\overline{\boldsymbol{x}}^{\prime}$, respectively. By the same discussion as in the proof of Proposition 2.1, we have $\tilde{\phi}\left(\overline{\boldsymbol{x}}^{\prime}\right)=\tilde{\phi}\left(\overline{\boldsymbol{x}}^{(i)}\right), i=1, \ldots, n$. Since $\phi$ is right $\epsilon$-resolving and $f$ is $\epsilon$-expansive, using a similar argument to one in that proof, we see that $\overline{\boldsymbol{x}}^{(i)}, i=1, \ldots, n$, are distinct. Since $n$ is the maximum number of inverses for $\tilde{\phi}$, we have $\overline{\boldsymbol{x}}^{\prime}=\overline{\boldsymbol{x}}^{(k)}$ for some $1 \leq k \leq n$. The same argument as in the proof of Proposition 2.1 shows that $\boldsymbol{x}^{\prime} \in V_{\epsilon}^{-}\left(\boldsymbol{x}^{(k)}, g\right)$, which is a contradiction.

Let $\boldsymbol{z}$ be a point of $\mathcal{O}_{g}$ which has the minimum number of inverses under $\tilde{\phi}$ among all points in $\bigcup_{j=0}^{\infty} \sigma_{g}^{j}\left(V_{\epsilon}^{-}\left(\sigma_{g}^{-j}(\boldsymbol{y}), g\right)\right)$. We may assume that $\boldsymbol{z} \in V_{\epsilon}^{-}(\boldsymbol{y}, g)$. (For if $\boldsymbol{z} \in \sigma_{g}^{J}\left(V_{\epsilon}^{-}\left(\sigma_{g}^{-J}(\boldsymbol{y}), g\right)\right.$ ) with $J \in \mathbf{N}$, then we may reuse $\boldsymbol{z}, \boldsymbol{y}$ and $\boldsymbol{x}^{(i)}, i=$ $1, \ldots, n$, to mean $\sigma_{g}^{-J}(\boldsymbol{z}), \sigma_{g}^{-J}(\boldsymbol{y})$ and $\sigma_{f}^{-J}\left(\boldsymbol{x}^{(i)}\right), i=1, \ldots, n$, respectively.) Let $\boldsymbol{w}^{(1)}, \ldots, \boldsymbol{w}^{(m)}$ be the inverses of $\boldsymbol{z}$ under $\tilde{\phi}$. Then, by the above, they are contained in $\bigcup_{i=1}^{n} V_{\epsilon}^{-}\left(\boldsymbol{x}^{(i)}, f\right)$. If there were $1 \leq l<l^{\prime} \leq m$ and $1 \leq i \leq n$ with $\boldsymbol{w}^{(l)}, \boldsymbol{w}^{\left(l^{\prime}\right)} \in$ $V_{\epsilon}^{-}\left(\boldsymbol{x}^{(i)}, f\right)$, then $\boldsymbol{w}^{(l)}$ and $\boldsymbol{w}^{\left(l^{\prime}\right)}$ would be left $\epsilon$-asymptotic, i.e., $d_{X}\left(w_{-j}^{(l)}, w_{-j}^{\left(l^{\prime}\right)}\right) \leq \epsilon$ for all sufficiently large $j$, because $f$ is $\epsilon$-expansive. Since $\tilde{\phi}\left(\boldsymbol{w}^{(l)}\right)=\tilde{\phi}\left(\boldsymbol{w}^{\left(l^{\prime}\right)}\right)$ and $\phi$ is right $\epsilon$-resolving, we would have $\boldsymbol{w}^{(l)}=\boldsymbol{w}^{\left(l^{\prime}\right)}$, which is not the case. Therefore we may assume that $\boldsymbol{w}^{(i)} \in V_{\epsilon}^{-}\left(\boldsymbol{x}^{(i)}, f\right), i=1, \ldots, m$. We claim that $m=n$.

Assume the contrary. Then there is no $\boldsymbol{u} \in V_{\epsilon}^{-}\left(\boldsymbol{x}^{(n)}, f\right)$ with $\tilde{\phi}(\boldsymbol{u})=\boldsymbol{z}$. Let $\delta>0$ be such that any $2 \delta$-pseudo-orbit of $f$ is $\epsilon$-traced by some orbit of $f$. Since $\mathcal{O}_{f}$ is compact, there is $0<\beta<\epsilon$ such that $\bigcup_{i=1}^{m} U_{\delta}\left(\boldsymbol{w}^{(i)}\right) \supset \tilde{\phi}^{-1}\left(V_{\beta}(\boldsymbol{z})\right)$, where $U_{\delta}\left(\boldsymbol{w}^{(i)}\right)$ and $V_{\beta}(\boldsymbol{z})$ denote the open ball with center $\boldsymbol{w}^{(i)}$ and radius $\delta$ and the closed ball with center $\boldsymbol{z}$ and radius $\beta$, respectively. For otherwise, there would be a point $\overline{\boldsymbol{w}} \notin \bigcup_{i=1}^{m} U_{\delta}\left(\boldsymbol{w}^{(i)}\right)$ with $\tilde{\phi}(\overline{\boldsymbol{w}})=\boldsymbol{z}$. Since $\mathcal{O}_{g}$ is compact and $g$ is $\beta$-expansive, it follows that there is an integer $K \geq 2$ such that, for any $\hat{\boldsymbol{z}} \in \mathcal{O}_{g}$, if $d_{Y}\left(z_{j}, \hat{z}_{j}\right) \leq \beta$ for $-K+1 \leq j \leq K-1$, then $d_{\mathcal{O}_{g}}(\boldsymbol{z}, \hat{\boldsymbol{z}}) \leq \beta$. Let $\alpha>0$ be such that any $\alpha$-pseudo-orbit of $g$ is $\beta$-traced by some orbit of $g$. Since $\boldsymbol{z} \in V_{\epsilon}^{-}(\boldsymbol{y}, g)$ and $g$ is $\epsilon$-expansive, there is $M>0$ such that $d_{Y}\left(y_{-M}, z_{-M}\right)<\alpha / 2$. Let $\gamma>0$ be such that $\operatorname{id}_{X}(\gamma, \alpha / 2)$-codes $\phi$ with $\gamma<\delta$. Since $f$ is topologically transitive, there are $s \in \mathcal{O}_{f}$ and $L \geq 1$ such that $d_{X}\left(s_{0}, w_{K}^{(1)}\right)<\gamma$ and $d_{X}\left(s_{L}, x_{-M}^{(n)}\right)<\gamma$. We have $d_{Y}\left(\phi\left(s_{0}\right), \phi\left(w_{K}^{(1)}\right)\right) \leq \alpha / 2$, and we also have $d_{Y}\left(\phi\left(s_{L}\right), z_{-M}\right) \leq \alpha$, because $d_{Y}\left(\phi\left(s_{L}\right), \phi\left(x_{-M}^{(n)}\right)\right)+d_{Y}\left(\phi\left(x_{-M}^{(n)}\right), z_{-M}\right)=d_{Y}\left(\phi\left(s_{L}\right), \phi\left(x_{-M}^{(n)}\right)\right)+d_{Y}\left(y_{-M}, z_{-M}\right) \leq$ $\alpha / 2+\alpha / 2$. We can define

$$
\hat{\boldsymbol{z}}=\operatorname{trace}_{\beta}\left(\boldsymbol{z}_{(-\infty, K) *} \stackrel{\alpha}{\circ} \tilde{\phi}(\boldsymbol{s})_{[0, L)} \stackrel{\alpha}{\circ} \boldsymbol{z}_{[-M, \infty)}\right) .
$$

We have $d_{Y}\left(z_{j}, \hat{z}_{j}\right) \leq \beta$ for $j \leq K-1$, so that $d_{Y}\left(\hat{z}_{j}, y_{j}\right) \leq \epsilon+\beta<2 \epsilon$ for $j \leq 0$. Since $g$ is $2 \epsilon$-expansive, there is $N \in \mathbf{N}$ such that $d_{Y}\left(\hat{z}_{j}, y_{j}\right) \leq \epsilon$ for $j \leq-N$. That is, $\hat{\boldsymbol{z}} \in \sigma_{g}^{N}\left(V_{\epsilon}^{-}\left(\sigma_{g}^{-N}(\boldsymbol{y}), g\right)\right)$. Let $\hat{\boldsymbol{w}}^{(1)}, \ldots, \hat{\boldsymbol{w}}^{(l)}$ be the inverses of $\hat{\boldsymbol{z}}$ under $\tilde{\phi}$. Then $m \leq l \leq n$, and

$$
\bigcup_{i=1}^{m} U_{\delta}\left(\boldsymbol{w}^{(i)}\right) \supset \tilde{\phi}^{-1}\left(V_{\beta}(\boldsymbol{z})\right) \supset \tilde{\phi}^{-1}(\hat{\boldsymbol{z}})=\left\{\hat{\boldsymbol{w}}^{(1)}, \ldots, \hat{\boldsymbol{w}}^{(l)}\right\} .
$$

If $U_{\delta}\left(\boldsymbol{w}^{(p)}\right) \ni \hat{\boldsymbol{w}}^{(q)}$ with $1 \leq p \leq m$ and $1 \leq q \leq l$, then we can define

$$
\boldsymbol{w}^{(p, q)}=\operatorname{trace}_{\epsilon}\left(\hat{\boldsymbol{w}}_{(-\infty, 1)^{*}}^{(q)} \stackrel{2 \delta}{\circ} \boldsymbol{w}_{[1, \infty)}^{(p)}\right) \in \mathcal{O}_{f}
$$


Since $d_{X}\left(w_{j}^{(p, q)}, \hat{w}_{j}^{(q)}\right) \leq \epsilon$ for $j<1$ and $\phi$ is $\epsilon$-preserving, $d_{Y}\left(\phi\left(w_{j}^{(p, q)}\right), \hat{z}_{j}\right) \leq \epsilon$ for $j<1$. Hence we have $d_{Y}\left(\phi\left(w_{j}^{(p, q)}\right), z_{j}\right) \leq \epsilon+\beta$ for $j<1$, because $d_{Y}\left(\hat{z}_{j}, z_{j}\right) \leq \beta$ for $j<1$. We also have $d_{Y}\left(\phi\left(w_{j}^{(p, q)}\right), z_{j}\right) \leq \epsilon$ for $j \geq 1$. Since $g$ is $(\epsilon+\beta)$-expansive, we have $\tilde{\phi}\left(\boldsymbol{w}^{(p, q)}\right)=\boldsymbol{z}$, so that $\boldsymbol{w}^{(p, q)}=\boldsymbol{w}^{(i)}$ for some $1 \leq i \leq m$. Therefore, by the definition of $\boldsymbol{w}^{(p, q)}, \hat{\boldsymbol{w}}^{(q)} \in V_{\epsilon}^{-}\left(\boldsymbol{w}^{(i)}, f\right)$ for some $1 \leq i \leq m$. If there were no $q$ with $\hat{\boldsymbol{w}}^{(q)} \in V_{\epsilon}^{-}\left(\boldsymbol{w}^{(1)}, f\right)$, then there would be distinct $q$ and $q^{\prime}$ and $2 \leq i \leq m$ such that $\hat{\boldsymbol{w}}^{(q)}, \hat{\boldsymbol{w}}^{\left(q^{\prime}\right)} \in V_{\epsilon}^{-}\left(\boldsymbol{w}^{(i)}, f\right)$, because $l \geq m$. This is not the case, as seen by the same discussion as above. Thus we may assume that $\hat{\boldsymbol{w}}^{(1)} \in V_{\epsilon}^{-}\left(\boldsymbol{w}^{(1)}, f\right)$. Since $\gamma<\delta$, we can define

$$
\boldsymbol{t}=\operatorname{trace}_{\epsilon}\left(\boldsymbol{w}_{(-\infty, K)^{*}}^{(1)} \stackrel{\delta}{\circ} \boldsymbol{s}_{[0, L)} \stackrel{\delta}{\circ} \boldsymbol{x}_{[-M, \infty)}^{(n)}\right) .
$$

Since $\phi$ is $\epsilon$-preserving and $g$ is $(\epsilon+\beta)$-expansive, it follows that

$$
\begin{aligned}
\tilde{\phi}(\boldsymbol{t}) & =\operatorname{trace}_{\beta}\left(\tilde{\phi}\left(\boldsymbol{w}^{(1)}\right)_{(-\infty, K)^{*}} \stackrel{\alpha}{\circ} \tilde{\phi}(\boldsymbol{s})_{[0, L)} \stackrel{\alpha}{\circ} \tilde{\phi}\left(\boldsymbol{x}^{(n)}\right)_{[-M, \infty)}\right) \\
& =\operatorname{trace}_{\beta}\left(\boldsymbol{z}_{(-\infty, K)^{*}} \stackrel{\alpha}{\circ} \tilde{\phi}(\boldsymbol{s})_{[0, L)} \stackrel{\alpha}{\circ} \boldsymbol{y}_{[-M, \infty)}\right) .
\end{aligned}
$$

Since

$$
\tilde{\phi}\left(\hat{\boldsymbol{w}}^{(1)}\right)=\hat{\boldsymbol{z}}=\operatorname{trace}_{\beta}\left(\boldsymbol{z}_{(-\infty, K)^{*}} \stackrel{\alpha}{\circ} \tilde{\phi}(\boldsymbol{s})_{[0, L)} \stackrel{\alpha}{\circ} \boldsymbol{z}_{[-M, \infty)}\right)
$$

and we can have chosen $\beta$ so that $2 \beta<\epsilon$ and $\operatorname{id}_{Y}(2 \beta, \epsilon)$-codes $g$, we have

$$
d_{Y}\left(\phi\left(t_{j}\right), \phi\left(\hat{w}_{j}^{(1)}\right)\right) \leq \epsilon \quad \text { for } \quad j \leq K+L .
$$

Since $\hat{\boldsymbol{w}}^{(1)}, \boldsymbol{t} \in V_{\epsilon}^{-}\left(\boldsymbol{w}^{(1)}, f\right)$ and $f$ is $\epsilon$-expansive, $\hat{\boldsymbol{w}}^{(1)}$ and $\boldsymbol{t}$ are left $\epsilon$-asymptotic. Therefore, since $\phi$ is right $\epsilon$-resolving, it follows that

$$
d_{X}\left(\hat{w}_{j}^{(1)}, t_{j}\right) \leq \epsilon \quad \text { for } \quad j \leq K+L .
$$

Since $d_{X}\left(t_{K+L}, x_{-M}^{(n)}\right) \leq \epsilon$ (by the definition of $\boldsymbol{t}$ ), we have $d_{X}\left(\hat{w}_{K+L}^{(1)}, x_{-M}^{(n)}\right) \leq 2 \epsilon<$ $\delta_{0}$. Thus we can define

$$
\boldsymbol{u}=\operatorname{trace}_{\epsilon_{0}}\left(\boldsymbol{x}_{(-\infty,-M)^{*}}^{(n)} \stackrel{\delta_{0}}{\circ} \hat{\boldsymbol{w}}_{[K+L, \infty)}^{(1)}\right) .
$$

We have $d_{Y}\left(\phi\left(u_{j}\right), z_{j}\right) \leq d_{Y}\left(\phi\left(u_{j}\right), \phi\left(x_{j}^{(n)}\right)\right)+d_{Y}\left(y_{j}, z_{j}\right) \leq c / 2+\epsilon$ for $j<-M$, since $\operatorname{id}_{X}\left(\epsilon_{0}, c / 2\right)$-codes $\phi$. Recalling the definition of $\hat{z}$, we also have $d_{Y}\left(\phi\left(u_{j}\right), z_{j}\right) \leq$ $d_{Y}\left(\phi\left(u_{j}\right), \phi\left(\hat{w}_{K+L+M+j}^{(1)}\right)\right)+d_{Y}\left(\hat{z}_{K+L+M+j}, z_{j}\right) \leq c / 2+\beta$ for $j \geq-M$. Hence we have $d_{Y}\left(\phi\left(u_{j}\right), z_{j}\right) \leq c / 2+c / 2=c$ for all $j \in \mathbf{Z}$. Thus we have $\tilde{\phi}(\boldsymbol{u})=\boldsymbol{z}$, because $g$ is $c$-expansive. Further we recall that $\tilde{\phi}\left(\boldsymbol{x}^{(n)}\right)=\boldsymbol{y}$ and $\boldsymbol{z} \in V_{\epsilon}^{-}(\boldsymbol{y}, g)$, and we note that $\boldsymbol{u}$ and $\boldsymbol{x}^{(n)}$ are left $\epsilon$-asymptotic. Therefore, since $\phi$ is right $\epsilon$-resolving, it follows that $\boldsymbol{u} \in V_{\epsilon}^{-}\left(\boldsymbol{x}^{(n)}, f\right)$, which is the desired contradiction. Thus we conclude that $m=n$.

Therefore it follows that $\tilde{\phi}\left(V_{\epsilon}^{-}\left(\boldsymbol{x}^{(i)}, f\right)\right)=V_{\epsilon}^{-}(\boldsymbol{y}, g)$ for $i=1, \ldots, n$. Putting $\boldsymbol{x}^{(1)}=\boldsymbol{w}$, we particularly have

$$
\tilde{\phi}\left(V_{\epsilon}^{-}(\boldsymbol{w}, f)\right)=V_{\epsilon}^{-}(\tilde{\phi}(\boldsymbol{w}), g) .
$$

Let $\boldsymbol{x} \in \mathcal{O}_{f}$. Let $\boldsymbol{z}^{\prime} \in V_{\epsilon}^{-}(\tilde{\phi}(\boldsymbol{x}), g)$. There is $M^{\prime}>0$ with $d_{Y}\left(\phi\left(x_{-M^{\prime}}\right), z_{-M^{\prime}}^{\prime}\right)<$ $\alpha / 2$. Let $K^{\prime}=1$. There are $\boldsymbol{s}^{\prime} \in \mathcal{O}_{f}$ and $L^{\prime} \geq 1$ such that $d_{X}\left(s_{0}^{\prime}, w_{K^{\prime}}\right)<\gamma$ and $d_{X}\left(s_{L^{\prime}}^{\prime}, x_{-M^{\prime}}\right)<\gamma$. Then, as we could define $\hat{\boldsymbol{z}}$ above, we can define

$$
\hat{\boldsymbol{z}}^{\prime}=\operatorname{trace}_{\beta}\left(\tilde{\phi}(\boldsymbol{w})_{\left(-\infty, K^{\prime}\right)^{*}} \stackrel{\alpha}{\circ} \tilde{\phi}\left(\boldsymbol{s}^{\prime}\right)_{\left[0, L^{\prime}\right)} \stackrel{\alpha}{\circ} \boldsymbol{z}_{\left[-M^{\prime}, \infty\right)}^{\prime}\right) .
$$


Then $\hat{\boldsymbol{z}}^{\prime} \in V_{\epsilon}^{-}(\tilde{\phi}(\boldsymbol{w}), g)$, and hence there is $\hat{\boldsymbol{w}} \in V_{\epsilon}^{-}(\boldsymbol{w}, f)$ with $\tilde{\phi}(\hat{\boldsymbol{w}})=\hat{\boldsymbol{z}}^{\prime}$. By the same arguments as above (consider $\boldsymbol{t}^{\prime}=\operatorname{trace}_{\epsilon}\left(\boldsymbol{w}_{\left(-\infty, K^{\prime}\right) *} \stackrel{\delta}{\circ} \boldsymbol{s}_{\left[0, L^{\prime}\right)}^{\prime} \stackrel{\delta}{\circ} \boldsymbol{x}_{\left[-M^{\prime}, \infty\right)}\right)$ ), we see that

$$
\boldsymbol{u}^{\prime}=\operatorname{trace}_{\epsilon_{0}}\left(\boldsymbol{x}_{\left(-\infty,-M^{\prime}\right) *} \stackrel{\delta_{0}}{\circ} \hat{\boldsymbol{w}}_{\left[K^{\prime}+L^{\prime}, \infty\right)}\right)
$$

can be defined, and that $\tilde{\phi}\left(\boldsymbol{u}^{\prime}\right)=\boldsymbol{z}^{\prime}$ and $\boldsymbol{u}^{\prime} \in V_{\epsilon}^{-}(\boldsymbol{x}, f)$.

Thus we have shown that, for each $\boldsymbol{x} \in \mathcal{O}_{f}, V_{\epsilon}^{-}(\phi(\boldsymbol{x}), g)=\tilde{\phi}\left(V_{\epsilon}^{-}(\boldsymbol{x}, f)\right)$, so that the proof of (1) is completed.

The proof of (2) is similar.

Lemma 2.3. Let $\epsilon>0$. Let $f: X \rightarrow X$ and $g: Y \rightarrow Y$ be $\epsilon$-expansive, onto maps with POTP with $g$ topologically transitive. Let $\delta>0$ be such that any $\delta$-pseudo-orbit of $f$ is $\epsilon$-traced by some orbit of $f$. Let $\phi:(X, f) \rightarrow(Y, g)$ be a both right $\epsilon$-closing and left $\epsilon$-closing, $\epsilon$-preserving, onto factor map.

(1) Any distinct orbits $\boldsymbol{x}$ and $\boldsymbol{x}^{\prime}$ of $f$ with $\tilde{\phi}(\boldsymbol{x})=\tilde{\phi}\left(\boldsymbol{x}^{\prime}\right)$ are totally $\delta$-separated, i.e., $d_{X}\left(x_{j}, x_{j}^{\prime}\right)>\delta$ for all $j \in \mathbf{Z}$.

(2) If $\phi$ is both right $\epsilon$-complete and left $\epsilon$-complete, then $\tilde{\phi}$ is constant-to-one and open.

Proof. (1) Let $\boldsymbol{x}, \boldsymbol{x}^{\prime} \in \mathcal{O}_{f}$ with $d_{X}\left(x_{0}, x_{0}^{\prime}\right) \leq \delta$ and $\tilde{\phi}(\boldsymbol{x})=\tilde{\phi}\left(\boldsymbol{x}^{\prime}\right)$. Then there is $\boldsymbol{w} \in V_{\epsilon}^{-}(\boldsymbol{x}, f) \cap V_{\epsilon}^{+}\left(\boldsymbol{x}^{\prime}, f\right)$. Since $\phi$ is $\epsilon$-preserving, we have $\tilde{\phi}(\boldsymbol{w}) \in V_{\epsilon}^{-}(\tilde{\phi}(\boldsymbol{x}), g) \cap$ $V_{\epsilon}^{+}\left(\tilde{\phi}\left(\boldsymbol{x}^{\prime}\right), g\right)$. Since $\tilde{\phi}(\boldsymbol{x})=\tilde{\phi}\left(\boldsymbol{x}^{\prime}\right)$ and $g$ is $\epsilon$-expansive, we have $\tilde{\phi}(\boldsymbol{w})=\tilde{\phi}(\boldsymbol{x})=$ $\tilde{\phi}\left(\boldsymbol{x}^{\prime}\right)$. Since $\phi$ is right $\epsilon$-closing, we have $\boldsymbol{w}=\boldsymbol{x}$, and since $\phi$ is left $\epsilon$-closing, we have $\boldsymbol{w}=\boldsymbol{x}^{\prime}$. Hence $\boldsymbol{x}=\boldsymbol{x}^{\prime}$. Thus (1) is proved.

(2) By Lemma 1.3 $\tilde{\phi}$ is onto, and from (1) it follows that $\tilde{\phi}$ is bounded-to-one. Let $\boldsymbol{y}, \boldsymbol{y}^{\prime} \in \mathcal{O}_{g}$. To prove that $\tilde{\phi}$ is constant-to-one, it suffices to show that $\#\left(\tilde{\phi}^{-1}(\boldsymbol{y})\right) \leq$ $\#\left(\tilde{\phi}^{-1}\left(\boldsymbol{y}^{\prime}\right)\right)$. Let $\gamma>0$ be such that any $\gamma$-pseudo-orbit of $g$ is $\epsilon$-traced by some orbit of $g$. Since $g$ is topologically transitive, there are $m \in \mathbf{N}$ and $z \in \mathcal{O}_{g}$ such that $d_{Y}\left(y_{1}, z_{0}\right)<\gamma$ and $d_{Y}\left(z_{m}, y_{0}^{\prime}\right)<\gamma$. Let $\hat{\boldsymbol{y}}=\operatorname{trace}_{\epsilon}\left(\boldsymbol{y}_{(-\infty, 1) *} \stackrel{\gamma}{\circ} \boldsymbol{z}_{[0, m)} \stackrel{\gamma}{\circ} \boldsymbol{y}_{[0, \infty)}^{\prime}\right)$. Then

$$
\hat{\boldsymbol{y}} \in V_{\epsilon}^{-}(\boldsymbol{y}, g) \quad \text { and } \quad \sigma_{g}^{m+1}(\hat{\boldsymbol{y}}) \in V_{\epsilon}^{+}\left(\boldsymbol{y}^{\prime}, g\right) .
$$

Let $\tilde{\phi}^{-1}(\boldsymbol{y})=\left\{\boldsymbol{x}^{(1)}, \ldots, \boldsymbol{x}^{(k)}\right\}$. Then we have

$$
V_{\epsilon}^{-}\left(\boldsymbol{x}^{(i)}, f\right) \cap V_{\epsilon}^{-}\left(\boldsymbol{x}^{(j)}, f\right)=\emptyset \text { for } 1 \leq i<j \leq k .
$$

For otherwise, since $f$ is $\epsilon$-expansive, it follows from Lemma 1.1 that there would be $n \in \mathbf{N}$ such that $d_{X}\left(x_{-n}^{(i)}, x_{-n}^{(j)}\right)<\delta$, which is not the case because $\boldsymbol{x}^{(i)}$ and $\boldsymbol{x}^{(j)}$ are totally $\delta$-separated, by (1). Since $\phi$ is right $\epsilon$-complete, there is $\hat{\boldsymbol{x}}^{(i)} \in V_{\epsilon}^{-}\left(\boldsymbol{x}^{(i)}, f\right)$ with $\tilde{\phi}\left(\hat{\boldsymbol{x}}^{(i)}\right)=\hat{\boldsymbol{y}}$ for $i=1, \ldots, k$. Thus we have

$$
\#\left(\tilde{\phi}^{-1}(\hat{\boldsymbol{y}})\right) \geq k=\# \tilde{\phi}^{-1}(\boldsymbol{y}) .
$$

Since $\boldsymbol{y}^{\prime} \in V_{\epsilon}^{+}\left(\sigma_{g}^{m+1}(\hat{\boldsymbol{y}}), g\right)$, an argument similar shows that

$$
\#\left(\tilde{\phi}^{-1}\left(\boldsymbol{y}^{\prime}\right)\right) \geq \#\left(\tilde{\phi}^{-1}\left(\sigma_{g}^{m+1}(\hat{\boldsymbol{y}})\right)\right) .
$$

Thus we have $\#\left(\tilde{\phi}^{-1}\left(\boldsymbol{y}^{\prime}\right)\right) \geq \# \tilde{\phi}^{-1}(\boldsymbol{y})$, as desired.

Using (1) and the fact that $\tilde{\phi}$ is constant-to-one, a similar argument to that in the proof of Theorem 16.3 of $[\mathrm{H}]$ proves that $\tilde{\phi}$ is open. 


\section{Commuting EXPAnsive Onto MAPS}

Throughout the remainder of this paper we assume the following. For a finite number of spaces $X_{i}, i=1, \ldots, n$, if we consider a subset $S$ of the Cartesian product $\prod_{i=1}^{n} X_{i}$ as a metric space, then $S$ is endowed with the max metric, that is, for $u=\left(x_{i}\right)_{1 \leq i \leq k}$ and $u^{\prime}=\left(x_{i}^{\prime}\right)_{1 \leq i \leq k}$ in $S, x_{i}, x_{i}^{\prime} \in X_{i}$

$$
d_{S}\left(u, u^{\prime}\right)=\max \left\{d_{X_{i}}\left(x_{i}, x_{i}^{\prime}\right) \mid i=1, \ldots, n\right\} .
$$

Let $f: X \rightarrow X$ be an onto map. Let $k \in \mathbf{N}$. We define the higher block system of order $k$ of the dynamical system $(X, f)$ to be the dynamical system $\left(X_{f}^{[k]}, f^{[k]}\right)$, where

$$
X_{f}^{[k]}=\left\{\left(f^{i}(x)\right)_{0 \leq i \leq k-1} \mid x \in X\right\},
$$

$d_{X_{f}^{[k]}}$ is the max metric, and $f^{[k]}$ is given by

$$
f^{[k]}\left(\left(f^{i}(x)\right)_{0 \leq i \leq k-1}\right)=\left(f^{i}(x)\right)_{1 \leq i \leq k}, \quad x \in X .
$$

It is clear that if $f$ is $c$-expansive [positively $c$-expansive] with $c>0$, then $f^{[k]}$ is $c$-expansive [positively $c$-expansive].

Lemma 3.1. Let $f: X \rightarrow X$ be a c-expansive onto map with $c>0$. Let $\epsilon_{0}, \delta_{0}$ be numbers such that $0<\delta_{0} \leq \epsilon_{0}<c / 3$ and any $\delta_{0}$-pseudo-orbit of $f$ is $\epsilon_{0}$-traced by some orbit of $f$. Let $k \in \mathbf{N}$. Then any $\delta_{0}$-pseudo-orbit of $f^{[k]}$ is $\epsilon_{0}$-traced by some orbit of $f^{[k]}$.

Proof. Let $\left(u_{j}\right)_{j \in \mathbf{Z}}$ be a $\delta_{0}$-pseudo-orbit of $f^{[k]}$. Then there is a sequence $\left(x_{j}\right)_{j \in \mathbf{Z}}$ of points $x_{j}$ of $X$ such that, for all $j \in \mathbf{Z}, u_{j}=\left(f^{i}\left(x_{j}\right)\right)_{0 \leq i \leq k-1}$ and

$$
\max \left\{d_{X}\left(f^{i}\left(x_{j}\right), f^{i-1}\left(x_{j+1}\right)\right) \mid i=1, \ldots, k\right\} \leq \delta_{0} .
$$

It follows that, for $i=0, \ldots, k-1,\left(f^{i}\left(x_{j}\right)\right)_{j \in \mathbf{Z}}$ is a $\delta_{0}$-pseudo-orbit of $f$, so that there is $\left(y_{j}^{(i)}\right)_{j \in \mathbf{Z}} \in \mathcal{O}_{f}$ which $\epsilon_{0}$-traces $\left(f^{i}\left(x_{j}\right)\right)_{j \in \mathbf{Z}}$. Hence, for all $j \in \mathbf{Z}$,

and hence

$$
d_{X}\left(y_{j}^{(i)}, f^{i}\left(x_{j}\right)\right) \leq \epsilon_{0}, \quad i=0, \ldots, k-1,
$$

$$
d_{X}\left(y_{j+1}^{(i-1)}, f^{i-1}\left(x_{j+1}\right)\right) \leq \epsilon_{0}, \quad i=1, \ldots, k .
$$

Therefore we have, for $i=1, \ldots, k-1, d_{X}\left(y_{j}^{(i)}, y_{j+1}^{(i-1)}\right) \leq 2 \epsilon_{0}+\delta_{0}<c$ for $j \in \mathbf{Z}$. Since $\left(y_{j}^{(i)}\right)_{j \in \mathbf{Z}} \in \mathcal{O}_{f}$ and $f$ is $c$-expansive, we have $y_{j}^{(i)}=y_{j+1}^{(i-1)}$ for $i=1, \ldots, k-1$ and $j \in$ $\mathbf{Z}$, so that we have $y_{j}^{(i)}=y_{j+i}^{(0)}$ for $i=0, \ldots, k-1$ and $j \in \mathbf{Z}$. Let $v_{j}=\left(y_{j+i}^{(0)}\right)_{0 \leq i \leq k-1}$ for $j \in \mathbf{Z}$. Then $\left(v_{j}\right)_{j \in \mathbf{Z}} \in \mathcal{O}_{f^{[k]}}$. Since $d_{X}\left(f^{i}\left(x_{j}\right), y_{j+i}^{(0)}\right)=d_{X}\left(f^{i}\left(x_{j}\right), y_{j}^{(i)}\right) \leq \epsilon_{0}$ for $i=0, \ldots, k-1$ and all $j \in \mathbf{Z}$, we have $d_{X_{f}^{[k]}}\left(u_{j}, v_{j}\right)=\max \left\{d_{X}\left(f^{i}\left(x_{j}\right), y_{j+i}^{(0)}\right) \mid i=\right.$ $0, \ldots, k-1\} \leq \epsilon_{0}$ for all $j \in \mathbf{Z}$, so that $\left(v_{j}\right)_{j \in \mathbf{Z}} \epsilon_{0}$-traces $\left(u_{j}\right)_{j \in \mathbf{Z}}$.

Lemma 3.2. Let $f: X \rightarrow X$ and $g: X \rightarrow X$ be commuting onto maps. Let $g$ be c-expansive with $c>0$. Let $k \in \mathbf{N}$. Let $g_{f}^{[k]}: X_{f}^{[k]} \rightarrow X_{f}^{[k]}$ be defined by

$$
g_{f}^{[k]}\left(\left(f^{j}(x)\right)_{0 \leq j \leq k-1}\right)=\left(f^{j} g(x)\right)_{0 \leq j \leq k-1}, \quad x \in X .
$$

Let $\epsilon_{0}, \delta_{0}>0$ be such that $\epsilon_{0}<c / 2, \operatorname{id}_{X}\left(\epsilon_{0}, c / 2\right)$-codes $f$, and any $\delta_{0}$-pseudo-orbit of $g$ is $\epsilon_{0}$-traced by some orbit of $g$. Then any $\delta_{0}$-pseudo-orbit of $g_{f}^{[k]}$ is $\epsilon_{0}$-traced by some orbit of $g_{f}^{[k]}$. 
Proof. We may assume that $k \geq 2$. Let $F(x)$ denote $\left(f^{j}(x)\right)_{0 \leq j \leq k-1}$ for $x \in X$. Any $\delta_{0}$-pseudo-orbit of $g_{f}^{[k]}$ is of the form $\left(F\left(x_{i}\right)\right)_{i \in \mathbf{Z}}$, where $\left(x_{i}\right)_{i \in \mathbf{Z}}$ is a sequence of points such that $d_{X}\left(g f^{j}\left(x_{i}\right), f^{j}\left(x_{i+1}\right)\right) \leq \delta_{0}$ for $i \in \mathbf{Z}$ and $j=0, \ldots, k-1$. Hence $\left(f^{j}\left(x_{i}\right)\right)_{i \in \mathbf{Z}}$ is an $\delta_{0}$-pseudo-orbit of $g$ for $j=0, \ldots, k-1$. Thus for each $j=0, \ldots, k-1$, there is an orbit $\left(y_{i}^{(j)}\right)_{i \in \mathbf{Z}}$ of $g$ which $\epsilon_{0}$-traces $\left(f^{j}\left(x_{i}\right)\right)_{i \in \mathbf{Z}}$, i.e., for each $i \in \mathbf{Z}, d_{X}\left(y_{i}^{(j)}, f^{j}\left(x_{i}\right)\right) \leq \epsilon_{0}$ for $j=0, \ldots, k-1$. Therefore, for $0 \leq j \leq k-2$,

$$
d_{X}\left(y_{i}^{(j+1)}, f\left(y_{i}^{(j)}\right)\right) \leq d_{X}\left(y_{i}^{(j+1)}, f^{j+1}\left(x_{i}\right)\right)+d_{X}\left(f\left(f^{j}\left(x_{i}\right)\right), f\left(y_{i}^{(j)}\right)\right) \leq \epsilon_{0}+\frac{c}{2}<c .
$$

Since $\left(f\left(y_{i}^{(j)}\right)\right)_{i \in \mathbf{Z}}$ is an orbit of $g$ and $g$ is $c$-expansive, we have $y_{i}^{(j+1)}=f\left(y_{i}^{(j)}\right)$ for $0 \leq j \leq k-2$. Hence $\left(F\left(y_{i}^{(0)}\right)\right)_{i \in \mathbf{Z}}$ is an orbit of $g_{f}^{[k]}$ which $\epsilon_{0}$-traces $\left(F\left(x_{i}\right)\right)_{i \in \mathbf{Z}}$.

Let $\epsilon>0$. We define an $\epsilon$-textile-orbit-system $T$ to be a quadruple of $\epsilon$-preserving onto maps

$$
i_{X}: X \rightarrow W, \quad t_{X}: X \rightarrow W, \quad p_{Y}: Y \rightarrow W, \quad q_{Y}: Y \rightarrow W
$$

such that $i_{X}$ and $p_{Y}$ are 1-1 and

$$
q_{Y} p_{Y}^{-1} t_{X} i_{X}^{-1}=t_{X} i_{X}^{-1} q_{Y} p_{Y}^{-1}
$$

We write $T=\left(i_{X}, t_{X}: X \rightarrow W ; p_{Y}, q_{Y}: Y \rightarrow W\right)$. We call $z=\left(y, y^{\prime}, x, x^{\prime}\right) \in$ $Y \times Y \times X \times X$ a square of $T$ if

$$
i_{X}(x)=p_{Y}(y), \quad q_{Y}(y)=i_{X}\left(x^{\prime}\right), \quad t_{X}(x)=p_{Y}\left(y^{\prime}\right), \quad \text { and } \quad q_{Y}\left(y^{\prime}\right)=t_{X}\left(x^{\prime}\right) .
$$

Let $Z$ be the set of all squares of $T$ and let it be endowed with the max metric. We call $Z$ the square space of $T$. We define projections $i_{Z}, t_{Z}, p_{Z}, q_{Z}$ as follows: for $z=\left(y, y^{\prime}, x, x^{\prime}\right) \in Z, i_{Z}(z)=y, t_{Z}(z)=y^{\prime}, p_{Z}(z)=x$, and $q_{Z}(z)=x^{\prime}$. Then all the projections are $\epsilon$-preserving, and the following diagram commutes:

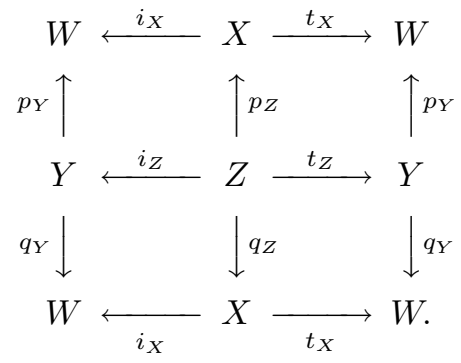

Since $i_{X}$ and $p_{Y}$ are 1-1, $i_{Z}$ and $p_{Z}$ are 1-1. A two-dimensional configuration $\left(z_{i j}\right)_{i, j \in \mathbf{Z}}$ of squares is called a textile-orbit of $T$ if for all $i, j \in \mathbf{Z}$,

$$
t_{Z}\left(z_{i, j-1}\right)=i_{Z}\left(z_{i j}\right) \text { and } q_{Z}\left(z_{i-1, j}\right)=p_{Z}\left(z_{i j}\right) .
$$

We say that $T$ is $L R$ if $\left\{t_{Z}, p_{Z}\right\}$ and $\left\{i_{Z}, q_{Z}\right\} \epsilon$-code each other. We also say that $T$ is $L L$ if $\left\{t_{Z}, p_{Z}\right\} \epsilon$-codes $\operatorname{id}_{Z}$ and $\left\{t_{Z}, q_{Z}\right\} \epsilon$-codes $\operatorname{id}_{Z}$.

Lemma 3.3. Let $\epsilon>0$. Let $T=\left(i_{X}, t_{X}: X \rightarrow W ; p_{Y}, q_{Y}: Y \rightarrow W\right)$ be an $\epsilon$ textile-orbit-system. Let $Z$ be the square space of $T$. Let $f=i_{Z}^{-1} t_{Z}$, let $f^{*}=p_{Z}^{-1} q_{Z}$, and let $g^{*}=p_{Y}^{-1} q_{Y}$. Assume that $g^{*}$ is topologically transitive and expansive and has POTP (and hence $f^{*}$ has the same properties, because $i_{Z}$ is a homeomorphism) and that there is a constant-triple $\left(c, \epsilon_{0}, \delta_{0}\right)$ for the factor map $t_{Z}:\left(Z, f^{*}\right) \rightarrow\left(Y, g^{*}\right)$ with $\epsilon<\delta_{0} / 2$. 
(1) If $T$ is $L R$, then, for any textile-orbits $\left(z_{i j}\right)_{i, j \in \mathbf{Z}}$ and $\left(z_{i j}^{\prime}\right)_{i, j \in \mathbf{Z}}$ of $T$, if

$$
V_{\epsilon}^{-}\left(\left(z_{i 0}\right)_{i \in \mathbf{Z}}, f^{*}\right) \cap V_{\epsilon}^{+}\left(\left(z_{i 0}^{\prime}\right)_{i \in \mathbf{Z}}, f^{*}\right) \ni \boldsymbol{z},
$$

then there is a textile-orbit $\left(\bar{z}_{i j}\right)_{i, j \in \mathbf{Z}}$ of $T$ such that $\left(\bar{z}_{i 0}\right)_{i \in \mathbf{Z}}=\boldsymbol{z}$,

$$
d_{Z}\left(\bar{z}_{i j}, z_{i j}\right) \leq \epsilon \forall i, j \leq 0, \quad \text { and } \quad d_{Z}\left(\bar{z}_{i j}, z_{i j}^{\prime}\right) \leq \epsilon \forall i, j \geq 0 .
$$

(2) If $T$ is $L L$, then the endomorphism $\tilde{f}$ of $\left(\mathcal{O}_{f^{*}}, \sigma_{f^{*}}\right)$ induced by $f:\left(Z, f^{*}\right) \rightarrow$ $\left(Z, f^{*}\right)$, is constant-to-one and open, and moreover for any textile-orbits $\left(z_{i j}\right)_{i, j \in \mathbf{Z}}$ and $\left(z_{i j}^{\prime}\right)_{i, j \in \mathbf{Z}}$ of $T$,

$$
\begin{aligned}
& V_{\epsilon}^{-}\left(\left(z_{i 0}\right)_{i \in \mathbf{Z}}, f^{*}\right) \cap V_{\epsilon}^{+}\left(\left(z_{i 0}^{\prime}\right)_{i \in \mathbf{Z}}, f^{*}\right) \neq \emptyset \\
& \quad \Rightarrow V_{2 \epsilon}^{-}\left(\left(z_{0 j}\right)_{j \in \mathbf{Z}}, f\right) \cap V_{0}^{+}\left(\left(z_{0 j}^{\prime}\right)_{j \in \mathbf{Z}}, f\right) \neq \emptyset .
\end{aligned}
$$

Proof. (1) Since $T$ is an LR $\epsilon$-textile-orbit-system, it follows that $i_{Z}$ is a left $\epsilon$ resolving conjugacy of $\left(Z, f^{*}\right)$ onto $\left(Y, g^{*}\right)$ and $t_{Z}$ is a right $\epsilon$-resolving factor map of $\left(Z, f^{*}\right)$ onto $\left(Y, g^{*}\right)$. From Proposition 2.1, Theorem 2.2, and the assumptions of the lemma, it follows that $i_{Z}$ is left $\epsilon$-complete and $t_{Z}$ is right $\epsilon$-complete.

Let $\left(z_{i j}\right)_{i, j \in \mathbf{Z}}$ and $\left(z_{i j}^{\prime}\right)_{i, j \in \mathbf{Z}}$ be textile-orbits of $T$, let $\boldsymbol{z} \in V_{\epsilon}^{-}\left(\left(z_{i 0}\right)_{i \in \mathbf{Z}}, f^{*}\right) \cap$ $V_{\epsilon}^{+}\left(\left(z_{i 0}^{\prime}\right)_{i \in \mathbf{Z}}, f^{*}\right)$, and put $\boldsymbol{z}=\left(z_{i}^{(0)}\right)_{i \in \mathbf{Z} \text {. }}$

Let $\left(y_{i j}\right)_{i \in \mathbf{Z}}=\left(i_{Z}\left(z_{i j}\right)\right)_{i \in \mathbf{Z}}$ for $j \in \mathbf{Z}$. Let $\left(y_{i}^{(0)}\right)_{i \in \mathbf{Z}}=\left(i_{Z}\left(z_{i}^{(0)}\right)\right)_{i \in \mathbf{Z}}$. Since $i_{Z}$ is $\epsilon$-preserving and $\left(z_{i}^{(0)}\right)_{i \in \mathbf{Z}} \in V_{\epsilon}^{-}\left(\left(z_{i 0}\right)_{i \in \mathbf{Z}}, f^{*}\right)$, we have $\left(y_{i}^{(0)}\right)_{i \in \mathbf{Z}} \in V_{\epsilon}^{-}\left(\left(y_{i 0}\right)_{i \in \mathbf{Z}}, g^{*}\right)$. Since $t_{Z}$ is right $\epsilon$-complete and $\left(y_{i 0}\right)_{i \in \mathbf{Z}}=\left(t_{Z}\left(z_{i,-1}\right)\right)_{i \in \mathbf{Z}}=\tilde{t}_{Z}\left(\left(z_{i,-1}\right)_{i \in \mathbf{Z}}\right)$, we have

$$
V_{\epsilon}^{-}\left(\left(y_{i 0}\right)_{i \in \mathbf{Z}}, g^{*}\right)=V_{\epsilon}^{-}\left(\tilde{t}_{Z}\left(\left(z_{i,-1}\right)_{i \in \mathbf{Z}}\right), g^{*}\right)=\tilde{t}_{Z}\left(V_{\epsilon}^{-}\left(\left(z_{i,-1}\right)_{i \in \mathbf{Z}}, f^{*}\right)\right) .
$$

Therefore, since $\left(y_{i}^{(0)}\right)_{i \in \mathbf{Z}} \in V_{\epsilon}^{-}\left(\left(y_{i 0}\right)_{i \in \mathbf{Z}}, g^{*}\right)$, there is

$$
\left(z_{i}^{(-1)}\right)_{i \in \mathbf{Z}} \in V_{\epsilon}^{-}\left(\left(z_{i,-1}\right)_{i \in \mathbf{Z}}, f^{*}\right)
$$

such that

$$
\tilde{t}_{Z}\left(\left(z_{i}^{(-1)}\right)_{i \in \mathbf{Z}}\right)=\left(y_{i}^{(0)}\right)_{i \in \mathbf{Z}}=\tilde{i}_{Z}\left(\left(z_{i}^{(0)}\right)_{i \in \mathbf{Z}}\right) .
$$

Let $\left(y_{i}^{(-1)}\right)_{i \in \mathbf{Z}}=\tilde{i}_{Z}\left(\left(z_{i}^{(-1)}\right)_{i \in \mathbf{Z}}\right)$. Then the same argument shows that there is

$$
\left(z_{i}^{(-2)}\right)_{i \in \mathbf{Z}} \in V_{\epsilon}^{-}\left(\left(z_{i,-2}\right)_{i \in \mathbf{Z}}, f^{*}\right)
$$

such that

$$
\tilde{t}_{Z}\left(\left(z_{i}^{(-2)}\right)_{i \in \mathbf{Z}}\right)=\left(y_{i}^{(-1)}\right)_{i \in \mathbf{Z}}=\tilde{i}_{Z}\left(\left(z_{i}^{(-1)}\right)_{i \in \mathbf{Z}) .}\right.
$$

Continuing in this way, we know that there are $\left(z_{i}^{(j)}\right)_{i \in \mathbf{Z}} \in \mathcal{O}_{f^{*}}, j \leq 0$, such that

$$
\left(z_{i}^{(j)}\right)_{i \in \mathbf{Z}} \in V_{\epsilon}^{-}\left(\left(z_{i j}\right)_{i \in \mathbf{Z}}, f^{*}\right) \text { and } \tilde{i}_{Z}\left(\left(z_{i}^{(j)}\right)_{i \in \mathbf{Z}}\right)=\tilde{t}_{Z}\left(\left(z_{i}^{(j-1)}\right)_{i \in \mathbf{Z})} \quad \text { for all } j \leq 0\right. \text {. }
$$

Furthermore, a similar argument, using the $\epsilon$-preserving property of $t_{Z}$ and the left $\epsilon$-completeness of $i_{Z}$, shows that there are $\left(z_{i}^{(j)}\right)_{i \in \mathbf{Z}} \in \mathcal{O}_{f^{*}}, j \geq 0$, such that

$$
\left(z_{i}^{(j)}\right)_{i \in \mathbf{Z}} \in V_{\epsilon}^{+}\left(\left(z_{i j}^{\prime}\right)_{i \in \mathbf{Z}}, f^{*}\right) \text { and } \tilde{t}_{Z}\left(\left(z_{i}^{(j)}\right)_{i \in \mathbf{Z}}\right)=\tilde{i}_{Z}\left(\left(z_{i}^{(j+1)}\right)_{i \in \mathbf{Z})} \text { for all } j \geq 0\right. \text {. }
$$

If we define $\left(\bar{z}_{i j}\right)_{i, j \in \mathbf{Z}}=\left(z_{i}^{(j)}\right)_{i, j \in \mathbf{Z}}$, then $\left(\bar{z}_{i j}\right)_{i, j \in \mathbf{Z}}$ is a textile-orbit of $T$ such that $\left(\bar{z}_{i 0}\right)_{i \in \mathbf{Z}}=\boldsymbol{z}, d_{Z}\left(\bar{z}_{i j}, z_{i j}\right) \leq \epsilon$ for all $i, j \leq 0$, and $d_{Z}\left(\bar{z}_{i j}, z_{i j}^{\prime}\right) \leq \epsilon$ for all $i, j \geq 0$.

(2) Since $T$ is an LL $\epsilon$-textile-orbit-system, it follows that $t_{Z}$ is a factor map of $\left(Z, f^{*}\right)$ onto $\left(Y, g^{*}\right)$ which is both left $\epsilon$-resolving and right $\epsilon$-resolving. Therefore, by Theorem 2.2, the assumptions of the lemma imply that $t_{Z}$ is both left $\epsilon$-complete and right $\epsilon$-complete. 
Since $t_{Z}$ is both left $\epsilon$-resolving and right $\epsilon$-resolving and $f^{*}$ is $\epsilon$-expansive, it is both left $\epsilon$-closing and right $\epsilon$-closing. Therefore it follows from Lemma 2.3 that $\tilde{t}_{Z}$ is constant-to-one and open, so that $\tilde{f}=\tilde{i}_{Z}^{-1} \tilde{t}_{Z}$ is constant-to-one and open.

Let $\left(z_{i j}\right)_{i, j \in \mathbf{Z}}$ and $\left(z_{i j}^{\prime}\right)_{i, j \in \mathbf{Z}}$ be textile-orbits of $T$ such that there is an orbit $\left(z_{i}^{(0)}\right)_{i \in \mathbf{Z}}$ of $f^{*}$ with

$$
\left(z_{i}^{(0)}\right)_{i \in \mathbf{Z}} \in V_{\epsilon}^{-}\left(\left(z_{i 0}\right)_{i \in \mathbf{Z}}, f^{*}\right) \cap V_{\epsilon}^{+}\left(\left(z_{i 0}^{\prime}\right)_{i \in \mathbf{Z}}, f^{*}\right) .
$$

The discussion using the $\epsilon$-preserving property of $i_{Z}$ and the right $\epsilon$-completeness of $t_{Z}$ in the proof of (1) has shown that there are $\left(z_{i}^{(j)}\right)_{i \in \mathbf{Z}} \in \mathcal{O}_{f^{*}}, j \leq 0$, such that

$$
\left(z_{i}^{(j)}\right)_{i \in \mathbf{Z}} \in V_{\epsilon}^{-}\left(\left(z_{i j}\right)_{i \in \mathbf{Z}}, f^{*}\right) \text { for all } j \leq 0
$$

and

$$
\tilde{i}_{Z}\left(\left(z_{i}^{(j)}\right)_{i \in \mathbf{Z}}\right)=\tilde{t}_{Z}\left(\left(z_{i}^{(j-1)}\right)_{i \in \mathbf{Z}}\right) \text { for all } j \leq 0 .
$$

By (3.2) we have

$$
d_{Z}\left(z_{0}^{(j)}, z_{0 j}\right) \leq \epsilon \text { for } j \leq 0
$$

By (3.1) we have

$$
\left(z_{i 0}^{\prime}\right)_{i \in \mathbf{Z}} \in V_{\epsilon}^{+}\left(\left(z_{i}^{(0)}\right)_{i \in \mathbf{Z}}, f^{*}\right)
$$

Let

$$
\left(\bar{z}_{i}^{(0)}\right)_{i \in \mathbf{Z}}=\left(z_{i 0}^{\prime}\right)_{i \in \mathbf{Z}}
$$

By using the $\epsilon$-preserving property of $i_{Z}$ and the left $\epsilon$-completeness of $t_{Z}$ together with (3.3), it is similarly proved that there are $\left(\bar{z}_{i}^{(j)}\right)_{i \in \mathbf{Z}} \in \mathcal{O}_{f^{*}}, j \leq 0$, such that for all $j \leq 0$

$$
\left(\bar{z}_{i}^{(j)}\right)_{i \in \mathbf{Z}} \in V_{\epsilon}^{+}\left(\left(z_{i}^{(j)}\right)_{i \in \mathbf{Z}}, f^{*}\right) \quad \text { and } \quad \tilde{i}_{Z}\left(\left(\bar{z}_{i}^{(j)}\right)_{i \in \mathbf{Z}}\right)=\tilde{t}_{Z}\left(\left(\bar{z}_{i}^{(j-1)}\right)_{i \in \mathbf{Z}) .}\right.
$$

Hence it follows that

$$
d_{Z}\left(\bar{z}_{0}^{(j)}, z_{0}^{(j)}\right) \leq \epsilon \text { for } j \leq 0
$$

and

$$
i_{Z}\left(\bar{z}_{0}^{(j)}\right)=t_{Z}\left(\bar{z}_{0}^{(j-1)}\right) \quad \text { for } j \leq 0 .
$$

Define $\hat{\boldsymbol{z}}=\left(\hat{z}_{j}\right)_{j \in \mathbf{Z}}$, where $\hat{z}_{j}=\bar{z}_{0}^{(j)}$ for $j \leq 0$ and $\hat{z}_{j}=z_{0 j}^{\prime}$ for $j \geq 0$. Then $\hat{\boldsymbol{z}} \in \mathcal{O}_{f}$ by (3.5) and (3.7), $d_{Z}\left(\hat{z}_{j}, z_{0 j}\right) \leq 2 \epsilon$ for $j \leq 0$ by (3.4) and (3.6), and $d_{Z}\left(\hat{z}_{j}, z_{0 j}^{\prime}\right)=0$ for $j \geq 0$. Thus we have $\hat{\boldsymbol{z}} \in V_{2 \epsilon}^{-}\left(\left(z_{0 j}\right)_{j \in \mathbf{Z}}, f\right) \cap V_{0}^{+}\left(\left(z_{0 j}^{\prime}\right)_{j \in \mathbf{Z}}, f\right)$.

Let $\varphi: X \rightarrow X$ and $\tau: X \rightarrow X$ be onto maps with $\varphi \tau=\tau \varphi$. Let $m, n \in \mathbf{N}$. Let $T_{m, n}(\varphi, \tau)$ be defined by

$$
\begin{aligned}
& T_{m, n}(\varphi, \tau)=\left\{i_{U}, t_{U}: U \rightarrow W ; p_{V}, q_{V}: V \rightarrow W\right\} ; \\
& U=\left\{\left(\varphi^{i} \tau^{j}(x)\right)_{0 \leq i \leq m-1,0 \leq j \leq n} \mid x \in X\right\}, \\
& V=\left\{\left(\varphi^{i} \tau^{j}(x)\right)_{0 \leq i \leq m, 0 \leq j \leq n-1} \mid x \in X\right\}, \\
& W=\left\{\left(\varphi^{i} \tau^{j}(x)\right)_{0 \leq i \leq m-1,0 \leq j \leq n-1} \mid x \in X\right\} ; \\
& \text { for } u=\left(\varphi^{i} \tau^{j}(x)\right)_{0 \leq i \leq m-1,0 \leq j \leq n} \in U, \\
& i_{U}(u)=\left(\varphi^{i} \tau^{j}(x)\right)_{0 \leq i \leq m-1,0 \leq j \leq n-1}, \quad t_{U}(u)=\left(\varphi^{i} \tau^{j}(x)\right)_{0 \leq i \leq m-1,1 \leq j \leq n} ;
\end{aligned}
$$


and for $v=\left(\varphi^{i} \tau^{j}(x)\right)_{0 \leq i \leq m, 0 \leq j \leq n-1} \in V$,

$$
p_{V}(v)=\left(\varphi^{i} \tau^{j}(x)\right)_{0 \leq i \leq m-1,0 \leq j \leq n-1}, \quad q_{V}(v)=\left(\varphi^{i} \tau^{j}(x)\right)_{1 \leq i \leq m, 0 \leq j \leq n-1} .
$$

It is clear that $i_{U}, t_{U}, p_{V}$, and $q_{V}$ are $\epsilon$-preserving onto maps for any $\epsilon>0$ and $i_{U}$ and $p_{V}$ are 1-1. Hence $T_{m, n}(\varphi, \tau)$ is an $\epsilon$-textile-orbit-system for any $\epsilon>0$. The square space of $T_{m, n}(\varphi, \tau)$ is given by

$$
Z=\left\{\left(\varphi^{i} \tau^{j}(x)\right)_{0 \leq i \leq m, 0 \leq j \leq n} \mid x \in X\right\} .
$$

Theorem 3.4. Let $\varphi: X \rightarrow X$ and $\tau: X \rightarrow X$ be positively expansive, onto maps with $\varphi \tau=\tau \varphi$. If $\varphi$ is topologically transitive and has POTP, then $\tau$ has POTP.

Proof. Since $\tau$ is expansive, if it has CC, then it has POTP (see, e.g., [AS or Hi]). Thus it suffices to show that for any $\epsilon>0$, there is $\delta>0$ such that for any $\boldsymbol{x}, \boldsymbol{x}^{\prime} \in \mathcal{O}_{\tau}$,

$$
d_{X}\left(x_{0}, x_{0}^{\prime}\right) \leq \delta \Rightarrow V_{\epsilon}^{-}(\boldsymbol{x}, \tau) \cap V_{\epsilon}^{+}\left(\boldsymbol{x}^{\prime}, \tau\right) \neq \emptyset .
$$

Let $\left(c, \epsilon_{0}, \delta_{0}\right)$ be a constant-triple for the factor map $\tau:(X, \varphi) \rightarrow(X, \varphi)$. We may assume that $\epsilon_{0}<c / 3$. Let $\epsilon$ be a number such that $0<\epsilon<\delta_{0} / 2$ and $\tau$ is positively $\epsilon$-expansive.

Since $\varphi$ and $\tau$ are positively $\epsilon$-expansive, it follows from Corollary 1.2 that there are $m, n \in \mathbf{N}$ such that $\left\{\varphi^{i} \mid 0 \leq i \leq m\right\} \epsilon$-codes $\tau$ and $\left\{\tau^{j} \mid 0 \leq j \leq n\right\}$ $\epsilon$-codes $\varphi$. We define an $\epsilon$-textile-orbit-system $T$ by $T=T_{m, n}(\varphi, \tau)$. It readily follows that $T$ is an LR $\epsilon$-textile-orbit-system. Let $f=i_{Z}^{-1} t_{Z}$, let $f^{*}=p_{Z}^{-1} q_{Z}$, and let $g^{*}=p_{V}^{-1} q_{V}$, where $Z$ is the square space of $T$. Since $\left(V, g^{*}\right)$ and $\left(Z, f^{*}\right)$ are conjugate to $(X, \varphi), f^{*}$ and $g^{*}$ are topologically transitive, expansive, onto maps with the same topological entropy and having POTP. We shall show that $\left(c, \epsilon_{0}, \delta_{0}\right)$ is a constant-triple for the factor map $t_{Z}:\left(Z, f^{*}\right) \rightarrow\left(V, g^{*}\right)$.

Since $\varphi$ is $c$-expansive, so are $f^{*}$ and $g^{*}$, by construction. Since any $\delta_{0}$-pseudoorbit of $\varphi$ is $\epsilon_{0}$-traced by some orbit of $\varphi$ with $0<\delta_{0} \leq \epsilon_{0}<c / 3$, it follows from Lemma 3.1 that any $\delta_{0}$-pseudo-orbit of $\varphi^{[m+1]}: X_{\varphi}^{[m+1]} \rightarrow X_{\varphi}^{[m+1]}$ is $\epsilon_{0}$-traced by some orbit of $\varphi^{[m+1]}$. If we define $\bar{\tau}: X_{\varphi}^{[m+1]} \rightarrow X_{\varphi}^{[m+1]}$ by

$$
\bar{\tau}\left(\left(\varphi^{i}(x)\right)_{0 \leq i \leq m}\right)=\left(\varphi^{i}(\tau(x))\right)_{0 \leq i \leq m}, \quad x \in X,
$$

then

$$
Z=\left\{\left(\bar{\tau}^{j}(y)\right)_{0 \leq j \leq n} \mid y \in X_{\varphi}^{[m+1]}\right\}=\left(X_{\varphi}^{[m+1]}\right)_{\bar{\tau}}^{[n+1]} .
$$

Since $\operatorname{id}_{X}\left(\epsilon_{0}, c / 2\right)$-codes $\tau$, it follows that $\operatorname{id}_{X_{\varphi}^{[m+1]}}\left(\epsilon_{0}, c / 2\right)$-codes $\bar{\tau}$. We note that $f^{*}$ sends $\left(\bar{\tau}^{j}(y)\right)_{0 \leq j \leq n}$ to $\left(\bar{\tau}^{j} \varphi^{[m+1]}(y)\right)_{0 \leq j \leq n}$ for $y \in X_{\varphi}^{[m+1]}$. Hence, by Lemma 3.2 , any $\delta_{0^{-}}$-pseudo-orbit of $f^{*}$ is $\epsilon_{0^{-}}$-traced by some orbit of $f^{*}$. Since $t_{Z}$ is $\epsilon_{0^{-}}$ preserving (by construction) and $\epsilon_{0}<c / 2, \mathrm{id}_{Z}\left(\epsilon_{0}, c / 2\right)$-codes $t_{Z}$. Thus we have shown that $\left(c, \epsilon_{0}, \delta_{0}\right)$ is a constant-triple for $t_{Z}:\left(Z, f^{*}\right) \rightarrow\left(V, g^{*}\right)$.

Since $f^{*}$ has POTP, there is $\delta_{1}>0$ such that any $\delta_{1}$-pseudo-orbit of $f^{*}$ is $\epsilon$-traced by some orbit of $f^{*}$. Let $\boldsymbol{z}, \boldsymbol{z}^{\prime} \in \mathcal{O}_{f}$ with $d_{Z}\left(z_{0}, z_{0}^{\prime}\right) \leq \delta_{1}$. There are textile-orbits $\left(z_{i j}\right)_{i, j \in \mathbf{Z}}$ and $\left(z_{i j}^{\prime}\right)_{i, j \in \mathbf{Z}}$ with $\left(z_{0 j}\right)_{j \in \mathbf{Z}}=\boldsymbol{z}$ and $\left(z_{0 j}^{\prime}\right)_{j \in \mathbf{Z}}=\boldsymbol{z}^{\prime}$. Since $d_{Z}\left(z_{00}, z_{00}^{\prime}\right)<\delta_{1}$, we have $V_{\epsilon}^{-}\left(\left(z_{i 0}\right)_{i \in \mathbf{Z}}, f^{*}\right) \cap V_{\epsilon}^{+}\left(\left(z_{i 0}^{\prime}\right)_{i \in \mathbf{Z}}, f^{*}\right) \neq \emptyset$. Hence, by Lemma 3.3 (1), we have $V_{\epsilon}^{-}(\boldsymbol{z}, f) \cap V_{\epsilon}^{+}\left(\boldsymbol{z}^{\prime}, f\right) \neq \emptyset$.

Let $z(x)=\left(\varphi^{i} \tau^{j}(x)\right)_{0 \leq i \leq m, 0 \leq j \leq n}$ for $x \in X$. Since $\varphi$ and $\tau$ are uniformly continuous, there is $\delta>0$ such that $\operatorname{id}_{X}\left(\delta, \delta_{1}\right)$-codes $z: X \rightarrow Z$. Hence it follows 
from the above that for any $\boldsymbol{x}, \boldsymbol{x}^{\prime} \in \mathcal{O}_{\tau}$, if $d_{X}\left(x_{0}, x_{0}^{\prime}\right)<\delta$, then $V_{\epsilon}^{-}\left(\left(z\left(x_{j}\right)\right)_{j \in \mathbf{Z}}, f\right) \cap$ $V_{\epsilon}^{+}\left(\left(z\left(x_{j}^{\prime}\right)\right)_{j \in \mathbf{Z}}, f\right) \neq \emptyset$, so that $V_{\epsilon}^{-}(\boldsymbol{x}, \tau) \cap V_{\epsilon}^{+}\left(\boldsymbol{x}^{\prime}, \tau\right) \neq \emptyset$.

Theorem 3.5. Let $\varphi: X \rightarrow X$ be a topologically transitive, expansive homeomorphism and $\tau: X \rightarrow X$ a positively expansive onto map. If $\varphi \tau=\tau \varphi$ and $\varphi$ has POTP, then $\tau$ has POTP and is constant-to-one.

Proof. Let $\epsilon>0$ be sufficiently small as in the proof of Theorem 3.4. Since $\tau$ is positively $\epsilon$-expansive, it follows from Corollary 1.2 that there is $n \in \mathbf{N}$ such that $\left\{\tau^{j} \mid j=0, \ldots, n\right\} \epsilon$-codes $\left\{\varphi, \varphi^{-1}\right\}$. Let $T$ be the $\epsilon$-textile-orbit-system defined by $T=T_{1, n}(\varphi, \tau)$ (see the paragraph before Theorem 3.4). Let $Z$ be the square space of $T$.

Since $\left\{\tau^{j} \mid j=0, \ldots, n\right\} \epsilon$-codes $\left\{\varphi, \varphi^{-1}\right\}$, it readily follows that $T$ is LL. Let $f$ and $f^{*}$ be defined in the same way as in the proof of Theorem 3.4. Then by Lemma $3.3(2), \tilde{f}$ is constant-to-one and open, and so is $f$, because $f^{*}$ is a homeomorphism (because $\varphi$ is a homeomorphism). Since $(X, \tau)$ and $(Z, f)$ are conjugate, $\tau$ is constant-to-one and open.

We give two proofs that $\tau$ has POTP. The first is given by appealing to the known result (see $[\mathrm{AH}]$ ) that a positively expansive onto map has POTP if (and only if) it is an open map. The second is given by showing that for any $\boldsymbol{x}, \boldsymbol{x}^{\prime} \in \mathcal{O}_{\tau}$, if $d_{X}\left(x_{0}, x_{0}^{\prime}\right)<\delta$, then $V_{2 \epsilon}^{-}(\boldsymbol{x}, \tau) \cap V_{0}^{+}\left(\boldsymbol{x}^{\prime}, \tau\right) \neq \emptyset$, by following the discussions in the proof of Theorem 3.4, letting $m=1$ and using Lemma 3.3(2) instead of Lemma $3.3(1)$.

We state a combination of Theorems 3.4 and 1.7.

Theorem 3.6. Let $\tau: X \rightarrow X$ be a topologically transitive, positively expansive onto map with POTP and with period $p$. Let $\varphi: X \rightarrow X$ be a positively expansive onto map with $\varphi \tau=\tau \varphi$. Then $\varphi$ has POTP and, for some $k$ dividing $p, X$ is partitioned into the $p / k$ basic sets $B_{i}$ of $\varphi$ such that the maps $\varphi \mid B_{i}$ have period $k$ and the systems $\left(B_{i}, \varphi \mid B_{i}\right)$ are a factor of each other.

Young-One Kim has conjectured the following refinement on Theorem 3.4, a special 0-dimensional case of which was proved by F. Blanchard and A. Maass BM]. The general 0-dimensional case of it has been proved by M. Boyle, D. Fiebig, and U. Fiebig $[\mathrm{BFF}]$.

Corollary 3.7. Let $\tau: X \rightarrow X$ be a topologically mixing, positively expansive onto map with POTP. Let $\varphi: X \rightarrow X$ be a positively expansive onto map with $\varphi \tau=\tau \varphi$. Then $\varphi$ has POTP and is topologically mixing.

We also state a combination of Theorems 3.5 and 1.7, and a combination of Theorems 3.5 and 1.6.

Theorem 3.8. Let $\tau: X \rightarrow X$ be a topologically transitive, expansive homeomorphism with POTP and period $p$. If $\varphi: X \rightarrow X$ is a positively expansive onto map with $\varphi \tau=\tau \varphi$, then $\varphi$ has POTP and, for some $k$ dividing $p, X$ is partitioned into the $p / k$ basic sets $B_{i}$ of $\varphi$ such that the maps $\varphi \mid B_{i}$ have period $k$ and are constant-to-one, and the systems $\left(B_{i}, \varphi \mid B_{i}\right)$ are conjugate to each other.

Theorem 3.9. Let $\tau: X \rightarrow X$ be an expansive homeomorphism with POTP. Let $\varphi: X \rightarrow X$ be a positively expansive onto map with $\varphi \tau=\tau \varphi$. If either one of $\tau$ and $\varphi$ is topologically mixing, then so is the other and $\varphi$ has POTP and is constant-to-one. 


\section{Essentially LR Endomorphisms}

Lemma 4.1. Let $\epsilon>0$. Let $T=\left(i_{X}, t_{X}: X \rightarrow W ; p_{Y}, q_{Y}: Y \rightarrow W\right)$ be an $L R \epsilon$ textile-orbit-system with $t_{X} 1-1$. Let $Z$ be the square space of $T$. Let $f=i_{Z}^{-1} t_{Z}$, let $f^{*}=p_{Z}^{-1} q_{Z}$, and let $g^{*}=p_{Y}^{-1} q_{Y}$ with $g^{*} \epsilon$-expansive. Then, for any textile-orbits $\left(z_{i j}\right)_{i, j \in \mathbf{Z}}$ and $\left(z_{i j}^{\prime}\right)_{i, j \in \mathbf{Z}}$ of $T$, if

$$
V_{\epsilon}^{-}\left(\left(z_{i 0}\right)_{i \in \mathbf{Z}}, f^{*}\right) \cap V_{\epsilon}^{+}\left(\left(z_{i 0}^{\prime}\right)_{i \in \mathbf{Z}}, f^{*}\right) \ni \boldsymbol{z},
$$

then there is a textile-orbit $\left(\bar{z}_{i j}\right)_{i, j \in \mathbf{Z}}$ of $T$ such that $\left(\bar{z}_{i 0}\right)_{i \in \mathbf{Z}}=\boldsymbol{z}$,

$$
d_{Z}\left(\bar{z}_{i j}, z_{i j}\right) \leq \epsilon \forall i, j \leq 0, \quad \text { and } \quad d_{Z}\left(\bar{z}_{i j}, z_{i j}^{\prime}\right) \leq \epsilon \forall i, j \geq 0 .
$$

Proof. Since $T$ is an LR $\epsilon$-textile-orbit-system and $t_{X}$ is a homeomorphism, it follows that $i_{Z}$ and $t_{Z}$ are respectively left $\epsilon$-resolving and right $\epsilon$-resolving conjugacies of $\left(Z, f^{*}\right)$ onto $\left(Y, g^{*}\right)$. Since $g^{*}$ is $\epsilon$-expansive, it follows from Proposition 2.1 that $i_{Z}$ is left $\epsilon$-complete and $t_{Z}$ is right $\epsilon$-complete. The remainder of the proof is the same as in the proof of Lemma 3.3(1).

Let $\epsilon>0$ and let $n \in \mathbf{N}$. We define the higher block system $T^{[n]}$ of order $n$ of an $\epsilon$-textile-orbit-system $T=\left(i_{X}, t_{X}: X \rightarrow W ; p_{Y}, q_{Y}: Y \rightarrow W\right)$ as follows. $T^{[1]}=T$. For $n \geq 2, T^{[n]}=\left(i_{X^{\prime}}, t_{X^{\prime}}: X^{\prime} \rightarrow W^{\prime} ; p_{Y^{\prime}}, q_{Y^{\prime}}: Y^{\prime} \rightarrow W^{\prime}\right)$, where, letting $g=i_{X}^{-1} t_{X}$ and $f=i_{Z}^{-1} t_{Z}$,

$$
\begin{aligned}
W^{\prime} & =\left\{\left(g^{j}(x)\right)_{0 \leq j \leq n-2} \mid x \in X\right\}, \quad X^{\prime}=\left\{\left(g^{j}(x)\right)_{0 \leq j \leq n-1} \mid x \in X\right\} \\
Y^{\prime} & =\left\{\left(f^{j}(z)\right)_{0 \leq j \leq n-2} \mid z \in Z\right\} ;
\end{aligned}
$$

for $x^{\prime}=\left(g^{j}(x)\right)_{0 \leq j \leq n-1}$ with $x \in X$,

$$
i_{X^{\prime}}\left(x^{\prime}\right)=\left(g^{j}(x)\right)_{0 \leq j \leq n-2} \quad \text { and } \quad t_{X^{\prime}}\left(x^{\prime}\right)=\left(g^{j}(x)\right)_{1 \leq j \leq n-1} ;
$$

and for $y^{\prime}=\left(f^{j}(z)\right)_{0 \leq j \leq n-2}$ with $z \in Z$,

$$
p_{Y^{\prime}}\left(y^{\prime}\right)=\left(p_{Z} f^{j}(z)\right)_{0 \leq j \leq n-2} \quad \text { and } \quad q_{Y^{\prime}}\left(y^{\prime}\right)=\left(q_{Z} f^{j}(z)\right)_{0 \leq j \leq n-2} .
$$

We easily see:

Remark 4.2. In the above notation, the following statements are valid.

(1) $T^{[n]}$ is an $\epsilon$-textile-orbit-system.

(2) If $T$ is LR, then so is $T^{[n]}$.

(3) If $g$ is $\epsilon$-expansive, then $g^{\prime}=i_{X^{\prime}}^{-1} t_{X^{\prime}}$ is $\epsilon$-expansive for all $n \geq 1$.

(4) There are homeomorphisms $\psi_{W}: W \rightarrow W^{\prime}, \psi_{X}: X \rightarrow X^{\prime}$ and $\psi_{Y}: Y \rightarrow Y^{\prime}$ such that $\psi_{W} i_{X}=i_{X^{\prime}} \psi_{X}, \quad \psi_{W} t_{X}=t_{X^{\prime}} \psi_{X}, \quad \psi_{W} p_{Y}=p_{Y^{\prime}} \psi_{Y}, \quad \psi_{W} q_{Y}=q_{Y^{\prime}} \psi_{Y}$.

We define the dual $T^{*}$ of $T=\left(i_{X}, t_{X}: X \rightarrow W ; p_{Y}, q_{Y}: Y \rightarrow W\right)$ by

$$
T^{*}=\left(i_{Y}, t_{Y}: Y \rightarrow W ; p_{X}, q_{X}: X \rightarrow W\right)
$$

with

$$
i_{Y}=p_{Y}, t_{Y}=q_{Y}, p_{X}=i_{X}, q_{X}=t_{X} .
$$

It is clear that if $T$ is an LR $\epsilon$-textile-orbit-system, then so is $T^{*}$.

Let $\tau: X \rightarrow X$ be an expansive onto map. An onto map $\varphi: X \rightarrow X$ is called an $L R$ endomorphism of $(X, \tau)$ if there are $\epsilon>0$ and an LR $\epsilon$-textileorbit-system $T=\left(i_{X}, t_{X}: X \rightarrow W ; p_{Y}, q_{Y}: Y \rightarrow W\right)$ such that $\tau=i_{X}^{-1} t_{X}$ and $\varphi=q_{Z} p_{Z}^{-1}$ with $\tau \epsilon$-expansive, where $Z$ is the square space of $T$. We define a positively LR endomorphism of $(X, \tau)$ by replacing 'with $\tau \epsilon$-expansive' by 'with $\tau$ positively $\epsilon$-expansive' in the preceding definition. We say that $\varphi$ is an essentially 
LR endomorphism of $(X, \tau)$ if there are an expansive onto map $\tau^{\prime}: X^{\prime} \rightarrow X^{\prime}$, an LR endomorphism of $\varphi^{\prime}$ of $\left(X^{\prime}, \tau^{\prime}\right)$, and a conjugacy $\psi:(X, \tau) \rightarrow\left(X^{\prime}, \tau^{\prime}\right)$ with $\varphi=\psi^{-1} \varphi^{\prime} \psi$. We define an essentially positively LR endomorphism similarly.

Theorem 4.3. Let $\tau: X \rightarrow X$ be an expansive homeomorphism and $\varphi$ an essentially LR automorphism of $(X, \tau)$.

(1) If $\tau$ has $C C$, then so does $\varphi$.

(2) If $\varphi$ is expansive and has $C C$, then $\tau$ has $C C$.

Proof. There are $\epsilon>0$, an LR $\epsilon$-textile-orbit-system $T=\left(i_{U}, t_{U}: U \rightarrow W ; p_{V}, q_{V}:\right.$ $V \rightarrow W)$ with $t_{U} 1-1$ and $g=i_{U}^{-1} t_{U} \epsilon$-expansive, and a conjugacy $\psi:(X, \tau) \rightarrow$ $(U, g)$ with $\psi^{-1} q_{Z} p_{Z}^{-1} \psi=\varphi$, where $Z$ is the square space of $T$. Since $\varphi$ is a bijection, so is $q_{Z}$. Let $f=i_{Z}^{-1} t_{Z}$ and let $f^{*}=p_{Z}^{-1} q_{Z}$. Since $g$ is $\epsilon$-expansive and $p_{Z}$ is an $\epsilon$-preserving bijection, it follows that $f$ is $\epsilon$-expansive.

(1) Assume that $\tau$ has CC. Since $\psi^{-1} p_{Z} f p_{Z}^{-1} \psi=\tau, f$ has CC. To prove that $\varphi$ has CC, it suffices to show that $f^{*}$ has $\mathrm{CC}$, because $\psi^{-1} p_{Z} f^{*} p_{Z}^{-1} \psi=\varphi$.

Let $\epsilon_{0}>0$. We shall show that there is $\delta_{0}>0$ such that, for any $\boldsymbol{z}, \boldsymbol{z}^{\prime} \in \mathcal{O}_{f^{*}}$, if $d_{Z}\left(z_{0}, z_{0}^{\prime}\right) \leq \delta_{0}$, then $V_{\epsilon_{0}}^{-}\left(\boldsymbol{z}, f^{*}\right) \cap V_{\epsilon_{0}}^{+}\left(\boldsymbol{z}^{\prime}, f^{*}\right) \neq \emptyset$. By Lemma 1.1, there is $n \in \mathbf{N}$ such that $\left\{f^{j} \mid-n \leq j \leq n\right\}\left(\epsilon, \epsilon_{0}\right)$-codes id $z$. Let $N=2 n+1$ and $\bar{T}=T^{[N]}$ with $\bar{T}=\left(i_{\bar{U}}, t_{\bar{U}}: \bar{U} \rightarrow \bar{W} ; p_{\bar{V}}, q_{\bar{V}}: \bar{V} \rightarrow \bar{W}\right)$. Then $\bar{T}$ is an LR $\epsilon$-textile-orbit-system such that $\bar{g}=i_{\bar{U}}^{-1} t_{\bar{U}}$ is $\epsilon$-expansive. Since $q_{Z}$ is $1-1$, so is $q_{\bar{V}}$. Let $\bar{Z}$ be the square space of $\bar{T}$. Let $\bar{f}=i_{\bar{Z}}^{-1} t_{\bar{Z}}$ and let $\bar{f}^{*}=p_{\bar{Z}}^{-1} q_{\bar{Z}}$. We note that $(\bar{Z}, \bar{f})$ is the higher block system of order $N$ of $(Z, f)$. Since $f$ has CC, so does $\bar{f}$. Hence there is $\delta>0$ such that, for any $\overline{\boldsymbol{z}}, \overline{\boldsymbol{z}}^{\prime} \in \mathcal{O}_{\bar{f}}$, if $d_{\bar{Z}}\left(\bar{z}_{0}, \bar{z}_{0}^{\prime}\right) \leq \delta$, then $V_{\epsilon}^{-}(\overline{\boldsymbol{z}}, \bar{f}) \cap V_{\epsilon}^{+}\left(\overline{\boldsymbol{z}}^{\prime}, \bar{f}\right) \neq \emptyset$. Let $\rho: Z \rightarrow \bar{Z}$ be the homeomorphism defined by $\rho(z)=\left(f^{j}(z)\right)_{-n \leq j \leq n}, z \in Z$. Let $\delta_{0}>0$ be such that $\operatorname{id}_{Z}\left(\delta_{0}, \delta\right)$-codes $\rho$.

Let $\boldsymbol{z}, \boldsymbol{z}^{\prime} \in \mathcal{O}_{f^{*}}$ with $d_{Z}\left(z_{0}, z_{0}^{\prime}\right) \leq \delta_{0}$. There are textile-orbits $\left(z_{i j}\right)_{i, j \in \mathbf{Z}}$ and $\left(z_{i j}^{\prime}\right)_{i, j \in \mathbf{Z}}$ of $T$ with $\left(z_{i 0}\right)_{i \in \mathbf{Z}}=\boldsymbol{z}$ and $\left(z_{i 0}^{\prime}\right)_{i \in \mathbf{Z}}=\boldsymbol{z}^{\prime}$. Then $\left(\rho\left(z_{i j}\right)\right)_{i, j \in \mathbf{Z}}$ and $\left(\rho\left(z_{i j}^{\prime}\right)\right)_{i, j \in \mathbf{Z}}$ are textile-orbits of $\bar{T}$. Since $d_{\bar{Z}}\left(\rho\left(z_{00}\right), \rho\left(z_{00}^{\prime}\right)\right) \leq \delta$, we have

$$
V_{\epsilon}^{-}\left(\left(\rho\left(z_{0 j}\right)\right)_{j \in \mathbf{Z}}, \bar{f}\right) \cap V_{\epsilon}^{+}\left(\left(\rho\left(z_{0 j}^{\prime}\right)\right)_{j \in \mathbf{Z}}, \bar{f}\right) \neq \emptyset .
$$

Hence, by the dual version of Lemma 4.1, we have

$$
V_{\epsilon}^{-}\left(\left(\rho\left(z_{i 0}\right)\right)_{i \in \mathbf{Z}}, \bar{f}^{*}\right) \cap V_{\epsilon}^{+}\left(\left(\rho\left(z_{i 0}^{\prime}\right)\right)_{i \in \mathbf{Z}}, \bar{f}^{*}\right) \neq \emptyset .
$$

Therefore, since $\rho\left(\epsilon, \epsilon_{0}\right)$-codes id $z$, we have $V_{\epsilon_{0}}^{-}\left(\boldsymbol{z}, f^{*}\right) \cap V_{\epsilon_{0}}^{+}\left(\boldsymbol{z}^{\prime}, f^{*}\right) \neq \emptyset$.

(2) We shall show that $\tau$ is an essentially LR automorphism of $(X, \varphi)$ assuming that $\varphi$ is expansive, because this and (1) prove (2). By the above, $\psi^{-1} g \psi=\tau$ and $\psi^{-1} \varphi^{\prime} \psi=\varphi$, where $\varphi^{\prime}=q_{Z} p_{Z}^{-1}$. Since $\varphi$ is expansive, so is $\varphi^{\prime}$. Let $\epsilon^{\prime}$ be an expansive constant for $\varphi^{\prime}$. Since $f$ is $\epsilon$-expansive, it follows from Lemma 1.1 that there is $k \geq 0$ such that $\left\{f^{j} \mid-k \leq j \leq k\right\}\left(\epsilon, \epsilon^{\prime}\right)$-codes $p_{Z}$. Let $\hat{T}=T^{[2 k+2]}$ with $\hat{T}=\left(i_{\hat{U}}, t_{\hat{U}}: \hat{U} \rightarrow \hat{W} ; p_{\hat{V}}, q_{\hat{V}}: \hat{V} \rightarrow \hat{W}\right)$. Then $\hat{T}$ is an LR $\epsilon$-textile-orbit-system. Let $\hat{\psi}: U \rightarrow \hat{V}$ be the homeomorphism defined by

$$
\hat{\psi}(u)=\left(f^{j} p_{Z}^{-1}(u)\right)_{-k \leq j \leq k}, \quad u \in U .
$$

Then $\hat{\psi}\left(\epsilon, \epsilon^{\prime}\right)$-codes $\operatorname{id}_{U}$. Let $\hat{g}^{*}=p_{\hat{V}}^{-1} q_{\hat{V}}$. Let $\hat{\varphi}^{*}=t_{\hat{Z}} i_{\hat{Z}}^{-1}$, where $\hat{Z}$ is the square space of $\hat{T}$. We have

$$
\hat{\psi} g \hat{\psi}^{-1}=\hat{\varphi}^{*} \quad \text { and } \quad \hat{\psi} \varphi^{\prime} \hat{\psi}^{-1}=\hat{g}^{*} .
$$


Since $\varphi^{\prime}$ is $\epsilon^{\prime}$-expansive, $\hat{g}^{*}$ is $\epsilon$-expansive (because $\hat{\psi}\left(\epsilon, \epsilon^{\prime}\right)$-codes $\operatorname{id}_{U}$ and $\hat{\psi}^{-1}$ is 1-1). Thus $\hat{\varphi}^{*}$ is an LR automorphism of $\left(\hat{V}, \hat{g}^{*}\right)$, so that $\tau$ is an essentially LR automorphism of $(X, \varphi)$.

As was mentioned in Introduction, Theorem 4.3 is closely related with the result of Boyle and Lind (Proposition 8.3 of $[\mathrm{BL}]$ ) that if one direction in an 'expansive component' of directions for a $\mathbf{Z}^{d}$ action is Markov, then so are all the directions of the component. For a 'Markov direction' is a generalized notion of an expansive homeomorphism with $\mathrm{CC}$, and it can be easily seen that if $\tau$ and $\varphi$ are expansive homeomorphisms of a space $X$ and $\varphi$ is an essentially LR automorphism of $(X, \tau)$, then the directions of $\tau$ and $\varphi$ are in the same expansive component of directions for the $\mathbf{Z}^{2}$-action generated by $\tau$ and $\varphi$. We note, however, that in Theorem 4.3(1), $\varphi$ is not assumed to be expansive. There are non-expansive LR automorphisms with CC of dynamical systems defined by expansive homeomorphisms with CC of 0-dimensional spaces (see Section 10 of [N1]).

Some results, including Theorem 4.3, on essentially LR automorphisms of expansive systems were announced without proofs (with somewhat different terminology) in N2]. Here we prove a strengthened version of another one of them, which gives a block-map-like characterization of the essentially LR automorphisms.

Proposition 4.4. Let $\tau: X \rightarrow X$ be an expansive onto map and $\varphi$ an automorphism of $(X, \tau)$.

(1) If $\varphi$ is an LR automorphism of $(X, \tau)$, then there is an expansive constant $\epsilon$ for $\tau$ such that $\varphi$ is of $(0, k)$ type and $\varphi^{-1}$ is of $(k, 0)$ type with respect to $\epsilon$ for some integer $k \geq 0$.

(2) If $\varphi$ is of $(0, k)$ type and $\varphi^{-1}$ is of $(k, 0)$ type with respect to an expansive constant $\epsilon$ for $\tau$ and with an integer $k \geq 0$, then $\varphi_{\tau}^{[k+1]}$ is an LR automorphism of $\left(X_{\tau}^{[k+1]}, \tau^{[k+1]}\right)$, where $\varphi_{\tau}^{[k+1]}: X_{\tau}^{[k+1]} \rightarrow X_{\tau}^{[k+1]}$ is defined by $\varphi_{\tau}^{[k+1]}\left(\left(\tau^{j}(x)\right)_{0 \leq j \leq k}\right)$ $=\left(\tau^{j} \varphi(x)\right)_{0 \leq j \leq k}, x \in X$.

Proof. (1) Since $\varphi$ is LR, there are an expansive constant $\epsilon$ for $\tau$ and an LR $\epsilon$ textile-orbit-system $T=\left(i_{X}, t_{X}: X \rightarrow W ; p_{Y}, q_{Y}: Y \rightarrow W\right)$ such that $\tau=i_{X}^{-1} t_{X}$, $\varphi=q_{Z} p_{Z}^{-1}$, and $q_{Z}$ is $1-1$, where $Z$ is the square space of $T$. Since $\tau$ is $\epsilon$-expansive, it follows from Lemma 1.1 that there is $m \geq 0$ such that $\left\{\tau^{0}, \ldots, \tau^{2 m}\right\} \epsilon$-codes $\left\{\tau_{Z}^{m} p_{Z}^{-1}, \tau_{Z}^{m} q_{Z}^{-1}\right\}$, where $\tau_{Z}=i_{Z}^{-1} t_{Z}$. Since $T$ is LR, $\left\{p_{Z}, t_{Z}\right\}$ and $\left\{i_{Z}, q_{Z}\right\} \epsilon$-code each other, so that

(*) for every $j \geq 0,\left\{p_{Z} \tau_{Z}^{j}, t_{Z} \tau_{Z}^{j}\right\}$ and $\left\{i_{Z} \tau_{Z}^{j}, q_{Z} \tau_{Z}^{j}\right\} \epsilon$-code each other.

Hence if $A=\left\{p_{Z} \tau_{Z}^{0}, \ldots, p_{Z} \tau_{Z}^{m}\right\} \cup\left\{t_{Z} \tau_{Z}^{m}\right\}$, then $A \epsilon$-codes

$$
\left\{p_{Z} \tau_{Z}^{0}, \ldots, p_{Z} \tau_{Z}^{m-1}, i_{Z} \tau_{Z}^{m}, q_{Z} \tau_{Z}^{m}\right\}=\left\{p_{Z} \tau_{Z}^{0}, \ldots, p_{Z} \tau_{Z}^{m-1}, t_{Z} \tau_{Z}^{m-1}, q_{Z} \tau_{Z}^{m}\right\} .
$$

This $\epsilon$-codes

$$
\begin{aligned}
\left\{p_{Z} \tau_{Z}^{0}, \ldots, p_{Z} \tau_{Z}^{m-2}, i_{Z} \tau_{Z}^{m-1}, q_{Z} \tau_{Z}^{m-1}, q_{Z} \tau_{Z}^{m}\right\} & \\
& =\left\{p_{Z} \tau_{Z}^{0}, \ldots, p_{Z} \tau_{Z}^{m-2}, t_{Z} \tau_{Z}^{m-2}, q_{Z} \tau_{Z}^{m-1}, q_{Z} \tau_{Z}^{m}\right\} .
\end{aligned}
$$

Continuing in this way, we finally see that $A \epsilon$-codes $\left\{i_{Z} \tau_{Z}^{0}\right\} \cup\left\{q_{Z} \tau_{Z}^{0}, \ldots, q_{Z} \tau_{Z}^{m}\right\}$, so that $A \epsilon$-codes $q_{Z}$. Hence $\left\{p_{Z} \tau_{Z}^{0} p_{Z}^{-1}, \ldots, p_{Z} \tau_{Z}^{m} p_{Z}^{-1}\right\} \cup\left\{t_{Z} \tau_{Z}^{m} p_{Z}^{-1}\right\} \epsilon$-codes $q_{Z} p_{Z}^{-1}$, namely, $\left\{\tau^{0}, \ldots, \tau^{m}\right\} \cup\left\{t_{Z} \tau_{Z}^{m} p_{Z}^{-1}\right\} \epsilon$-codes $\varphi$. Since $\left\{\tau^{0}, \ldots, \tau^{2 m}\right\} \epsilon$-codes $\tau_{Z}^{m} p_{Z}^{-1}$ and $t_{Z}$ is $\epsilon$-preserving, $\left\{\tau^{0}, \ldots, \tau^{2 m}\right\} \epsilon$-codes $t_{Z} \tau_{Z}^{m} p_{Z}^{-1}$. Thus $\left\{\tau^{0}, \ldots, \tau^{2 m}\right\} \epsilon$-codes $\varphi$. 
By $(*)$, we similarly see that $\left\{i_{Z}\right\} \cup\left\{q_{Z} \tau_{Z}^{0}, \ldots, q_{Z} \tau_{Z}^{m}\right\} \epsilon$-codes $\left\{p_{Z} \tau_{Z}^{0}, \ldots, p_{Z} \tau_{Z}^{m}\right\} \cup$ $\left\{t_{Z} \tau_{Z}^{m}\right\}$, so that it $\epsilon$-codes $p_{Z} \tau_{Z}^{m}$. Hence $\left\{i_{Z} q_{Z}^{-1}\right\} \cup\left\{q_{Z} \tau_{Z}^{0} q_{Z}^{-1}, \ldots, q_{Z} \tau_{Z}^{m} q_{Z}^{-1}\right\} \epsilon$-codes $p_{Z} \tau_{Z}^{m} q_{Z}^{-1}$, namely, $\left\{i_{Z} q_{Z}^{-1}\right\} \cup\left\{\tau^{0}, \ldots, \tau^{m}\right\} \epsilon$-codes $\varphi^{-1} \tau^{m}$, so that $\left\{i_{Z} q_{Z}^{-1} \tau^{m}\right\} \cup$ $\left\{\tau^{m}, \ldots, \tau^{2 m}\right\} \epsilon$-codes $\varphi^{-1} \tau^{2 m}$. Since $\left\{\tau^{0}, \ldots, \tau^{2 m}\right\} \epsilon$-codes $\tau_{Z}^{m} q_{Z}^{-1}$ and $i_{Z}$ is $\epsilon$ preserving, it follows that $\left\{\tau^{0}, \ldots, \tau^{2 m}\right\} \epsilon$-codes $i_{Z} q_{Z}^{-1} \tau^{m}$. Thus $\left\{\tau^{0}, \ldots, \tau^{2 m}\right\} \epsilon$ codes $\varphi^{-1} \tau^{2 m}$.

If we let $k=2 m$, then the conclusion of (1) is obtained.

(2) Let $T=\left(i_{U}, t_{U}: U \rightarrow W ; p_{V}, q_{V}: V \rightarrow W\right)$ be defined to be the $\epsilon$ textile-orbit-system $T_{1, k}(\varphi, \tau)$, the definition of which was given before Theorem 3.4. Clearly $i_{U}^{-1} t_{U}=\tau^{[k+1]}$ and $q_{Z} p_{Z}^{-1}=\varphi_{\tau}^{[k+1]}$, where $Z$ is the square space of $T$. It is also clear that $\tau^{[k+1]}$ is $\epsilon$-expansive. Since $\varphi$ is of $(0, k)$ type and $\varphi^{-1}$ is of $(k, 0)$ type with respect to $\epsilon$, it follows that $T$ is LR. Hence $\varphi_{\tau}^{[k+1]}$ is an LR automorphism of $\left(X_{\tau}^{[k+1]}, \tau^{[k+1]}\right)$.

Corollary 4.5. Let $\tau: X \rightarrow X$ be an expansive homeomorphism and $\varphi$ an automorphism of $(X, \tau)$. Then for all sufficiently large $n, \varphi \tau^{n}$ is an essentially $L R$ automorphism of $(X, \tau)$.

Proof. This follows from Corollary 1.2 and Proposition 4.4(2).

Proposition 4.6. Let $\tau: X \rightarrow X$ be an expansive onto map and $\varphi$ an essentially LR endomorphism of $(X, \tau)$. Then $\tau, \mathrm{id}_{X}$, and $\varphi^{m}, m \geq 1$, are essentially LR endomorphisms of $(X, \tau)$, and $\varphi \tau$ is an expansive, essentially LR endomorphism of $(X, \tau)$.

Proof. (i) First we prove that $\tau^{n}$ is an essentially LR endomorphism of $(X, \tau)$ for $n \geq 0$. Let $T=\left(i_{U}, t_{U}: U \rightarrow X ; p_{V}, q_{V}: V \rightarrow X\right)$ be defined as follows: $U=$ $\{(x, \tau(x)) \mid x \in X\}, V=\left\{\left(\tau^{i}(x)\right)_{0 \leq i \leq n} \mid x \in X\right\}$; for $u=(x, \tau(x)) \in U, i_{U}(u)=x$ and $t_{U}(u)=\tau(x)$; for $v=\left(\tau^{i}(x)\right)_{0<i<n} \in V, p_{V}(v)=x$ and $q_{V}(v)=\tau^{n}(x)$. Then it is clear that $T$ is an LR $\epsilon$-textile-orbit-system for any $\epsilon>0$. We have $i_{U}^{-1} t_{U}=\tau^{[2]}$, and $q_{Z} p_{Z}^{-1}=\left(\tau^{[2]}\right)^{n}$, where $Z$ is the square space of $T$. Let $\epsilon$ be an expansive constant for $\tau$. Then $\tau^{[2]}$ is $\epsilon$-expansive. Hence $\left(\tau^{[2]}\right)^{n}$ is an LR endomorphism of $\left(X_{\tau}^{[2]}, \tau^{[2]}\right)$, so that $\tau^{n}$ is an essentially LR endomorphism of $(X, \tau)$.

(ii) Let $\epsilon>0$. Let $T=\left(i_{X}, t_{X}: X \rightarrow W ; p_{Y}, q_{Y}: Y \rightarrow W\right)$ be an LR $\epsilon$ textile-orbit-system. Let $\tau_{X}=i_{X}^{-1} t_{X}$ with $\tau_{X} \epsilon$-expansive, let $\varphi_{Y}=p_{Y}^{-1} q_{Y}$, and let $\varphi_{X}=q_{Z} p_{Z}^{-1}$, where $Z$ is the square space of $T$. It suffices to show that the $\varphi_{X}^{m}, m \geq 1$, are LR endomorphisms of $\left(X, \tau_{X}\right)$ and that $\varphi_{X} \tau_{X}$ is an expansive, LR endomorphism of $\left(X, \tau_{X}\right)$.

(a) Let $m \geq 1$. Let $\hat{V}, p_{\hat{V}}: \hat{V} \rightarrow W$, and $q_{\hat{V}}: \hat{V} \rightarrow W$ be defined as follows: $\hat{V}=\left\{\left(\varphi_{Y}^{i}(y)\right)_{0 \leq i<m} \mid y \in Y\right\}$, and for $v=\left(\varphi_{Y}^{i}(y)\right)_{0 \leq i<m}$ let $p_{\hat{V}}(v)=p_{Y}(y)$ and $q_{\hat{V}}(v)=q_{Y} \varphi_{Y}^{m-1}(y)$. Let $\hat{T}=\left(i_{X}, t_{X}: X \rightarrow W ; p_{\hat{V}}, q_{\hat{V}}: \hat{V} \rightarrow W\right)$. Then $\hat{T}$ is an $\epsilon$-textile-orbit-system such that $i_{X}^{-1} t_{X}=\tau_{X}$ is $\epsilon$-expansive and $q_{\hat{Z}} p_{\hat{Z}}^{-1}=\varphi_{X}^{m}$, where $\hat{Z}$ is the square-space of $\hat{T}$. Since $T$ is LR, it follows that $\hat{T}$ is LR. Thus $\varphi_{X}^{m}$ is an LR endomorphism of $\left(X, \tau_{X}\right)$.

(b) Let $\tilde{p}: Z \rightarrow W$ and $\tilde{q}: Z \rightarrow W$ be defined as follows: for $z \in Z, \tilde{p}(z)=$ $p_{Y}\left(i_{Z}(z)\right)$ and $\tilde{q}(z)=q_{Y}\left(t_{Z}(z)\right)$. Let $\tilde{T}=\left(i_{X}, t_{X}: X \rightarrow W ; \tilde{p}, \tilde{q}: Z \rightarrow W\right)$. Then $\tilde{T}$ is an $\epsilon$-textile-orbit-system such that $i_{X}^{-1} t_{X}=\tau_{X}$ is $\epsilon$-expansive and $q_{\tilde{Z}} p_{\tilde{Z}}^{-1}=\varphi_{X} \tau_{X}$, where $\tilde{Z}$ is the square-space of $\tilde{T}$. Since $T$ is LR, it easily follows that $\tilde{T}$ is LR. Thus $\varphi_{X} \tau_{X}$ is an LR endomorphism of $\left(X, \tau_{X}\right)$. 
There is $\delta>0$ such that $\operatorname{id}_{X}(\delta, \epsilon)$-codes $p_{Z}^{-1}$. Let $\left(x_{k}\right)_{k \in \mathbf{Z}}$ and $\left(x_{k}^{\prime}\right)_{k \in \mathbf{Z}}$ be orbits of $\varphi_{X} \tau_{X}$ such that $d_{X}\left(x_{k}, x_{k}^{\prime}\right) \leq \delta$ for $k \in \mathbf{Z}$. There are textile-orbits $\left(z_{i j}\right)_{i, j \in \mathbf{Z}}$ and $\left(z_{i j}^{\prime}\right)_{i, j \in \mathbf{Z}}$ of $T$ such that $p_{Z}^{-1}\left(x_{k}\right)=z_{k k}$ and $p_{Z}^{-1}\left(x_{k}^{\prime}\right)=z_{k k}^{\prime}$ for $k \in \mathbf{Z}$. We have

$$
d_{Z}\left(z_{k k}, z_{k k}^{\prime}\right) \leq \epsilon \quad \text { for all } k \in \mathbf{Z} \text {. }
$$

Let $\tau_{Z}=i_{Z}^{-1} t_{Z}$ and let $\varphi_{Z}=p_{Z}^{-1} q_{Z}$. Since $T$ is LR, it follows that $\left\{\operatorname{id}_{Z}, \varphi_{Z} \tau_{Z}\right\}$ $\epsilon$-codes $\left\{\tau_{Z}, \varphi_{Z}\right\}$. By using repeatedly the fact that $\left\{\operatorname{id}_{Z}, \varphi_{Z} \tau_{Z}\right\} \epsilon$-codes $\varphi_{Z}$, and by induction on $i$, it is derived from $(*)$ that for all $i \geq 0, d_{Z}\left(z_{k+i, k}, z_{k+i, k}^{\prime}\right) \leq \epsilon$ for all $k \in \mathbf{Z}$. By using repeatedly the fact that $\left\{\operatorname{id}_{Z}, \varphi_{Z} \tau_{Z}\right\} \epsilon$-codes $\tau_{Z}$, and by induction on $j$, it is derived from $(*)$ that for all $j \geq 0, d_{Z}\left(z_{k, k+j}, z_{k, k+j}^{\prime}\right) \leq \epsilon$ for all $k \in \mathbf{Z}$. Thus $\left.d_{Z}\left(z_{i j}, z_{i j}^{\prime}\right)\right) \leq \epsilon$ for all $i, j \in \mathbf{Z}$. Therefore $d_{X}\left(p_{Z}\left(z_{i j}\right), p_{Z}\left(z_{i j}^{\prime}\right)\right) \leq \epsilon$ for all $i, j \in \mathbf{Z}$. Since $\tau_{X}$ is $\epsilon$-expansive and $\left(p_{Z}\left(z_{i j}\right)\right)_{j \in \mathbf{Z}}$ and $\left(p_{Z}\left(z_{i j}^{\prime}\right)\right)_{j \in \mathbf{Z}}$ are orbits of $\tau_{X}$ for $i \in \mathbf{Z}$, we have $p_{Z}\left(z_{i j}\right)=p_{Z}\left(z_{i j}^{\prime}\right)$ for all $i, j \in \mathbf{Z}$, so that $\left(x_{k}\right)_{k \in \mathbf{Z}}=\left(x_{k}^{\prime}\right)_{k \in \mathbf{Z}}$. Thus $\varphi_{X} \tau_{X}$ is $\delta$-expansive.

Proposition 4.7. Let $\tau: X \rightarrow X$ be a positively expansive onto map and $\varphi$ an onto endomorphism of $(X, \tau)$.

(1) If $\varphi$ is an essentially positively $L R$ endomorphism of $(X, \tau)$, then $\tau$, $\mathrm{id}_{X}$, and $\varphi^{m}, m \geq 1$, are essentially positively $L R$ endomorphisms of $(X, \tau)$, and $\varphi \tau$ is a positively expansive endomorphism of $(X, \tau)$.

(2) If $\varphi$ is positively expansive, then $\varphi$ is an essentially positively LR endomorphism of $(X, \tau)$.

Proof. The proof of Proposition 4.6 with minor modifications proves (1).

To prove (2), assume that $\tau$ and $\varphi$ are positively $\epsilon$-expansive with $\epsilon>0$. Then the LR $\epsilon$-textile-orbit-system in the proof of Theorem 3.4 shows that $\varphi$ is an essentially positively LR endomorphism of $(X, \tau)$.

Corollary 4.8. If $\tau: X \rightarrow X$ and $\varphi: X \rightarrow X$ are commuting positively expansive onto maps, then $\tau \varphi$ is positively expansive.

Let $\tau: X \rightarrow X$ and $\bar{\tau}: \bar{X} \rightarrow \bar{X}$ be onto maps. A factor map $\phi:(X, \tau) \rightarrow(\bar{X}, \bar{\tau})$ is said to be right closing if for any $\boldsymbol{x}, \boldsymbol{y} \in \mathcal{O}_{\tau}, \lim _{n \rightarrow \infty} d_{X}\left(x_{-n}, y_{-n}\right)=0$ and $\tilde{\phi}(\boldsymbol{x})=\tilde{\phi}(\boldsymbol{y})$ imply $\boldsymbol{x}=\boldsymbol{y}$.

The property of being right closing is an invariant for topological conjugacy between factor maps, which is defined as follows. Let $\tau_{i}: X_{i} \rightarrow X_{i}$ and $\bar{\tau}_{i}$ : $\bar{X}_{i} \rightarrow \bar{X}_{i}$ be onto maps for $i=1,2$. Factor maps $\phi_{1}:\left(X_{1}, \tau_{1}\right) \rightarrow\left(\bar{X}_{1}, \bar{\tau}_{1}\right)$ and $\phi_{2}:\left(X_{2}, \tau_{2}\right) \rightarrow\left(\bar{X}_{2}, \bar{\tau}_{2}\right)$ are said to be topologically conjugate if there are conjugacies $\psi:\left(X_{1}, \tau_{1}\right) \rightarrow\left(X_{2}, \tau_{2}\right)$ and $\bar{\psi}:\left(\bar{X}_{1}, \bar{\tau}_{1}\right) \rightarrow\left(\bar{X}_{2}, \bar{\tau}_{2}\right)$ with $\bar{\psi} \phi_{1}=\phi_{2} \psi$.

Remark 4.9. Let $\tau: X \rightarrow X$ be an expansive onto map. If $\varphi$ is an essentially LR endomorphism of $(X, \tau)$, then $\varphi$ is right closing.

Proof. Since the property of being right closing is an invariant for topological conjugacy between endomorphisms, it suffices to prove the remark for the case that $\varphi$ is an LR endomorphism of $(X, \tau)$. Let $\epsilon>0$ and let $T=\left(i_{X}, t_{X}: X \rightarrow W ; p_{Y}, q_{Y}\right.$ : $Y \rightarrow W)$ be an LR $\epsilon$-textile-orbit-system such that $\tau=i_{X}^{-1} t_{X}$ and $\varphi=q_{Z} p_{Z}^{-1}$ with $\tau$ e-expansive, where $Z$ is the square space of $T$. Let $f=i_{Z}^{-1} t_{Z}$. Since $T$ is LR, it follows that $q_{Z}:(Z, f) \rightarrow(X, \tau)$ is right $\epsilon$-resolving.

Let $\boldsymbol{x}, \boldsymbol{x}^{\prime} \in \mathcal{O}_{\tau}$ with $\lim _{n \rightarrow \infty} d_{X}\left(x_{-n}, x_{-n}^{\prime}\right)=0$ and $\varphi\left(x_{j}\right)=\varphi\left(x_{j}^{\prime}\right)$ for $j \in$ Z. Then there is $J \in \mathbf{Z}$ such that $d_{Z}\left(p_{Z}^{-1}\left(x_{j}\right), p_{Z}^{-1}\left(x_{j}^{\prime}\right)\right) \leq \epsilon$ for $j \leq J$. Since 
$q_{Z}\left(p_{Z}^{-1}(x)\right)=q_{Z}\left(p_{Z}^{-1}\left(x^{\prime}\right)\right)$ for all $j \in \mathbf{Z}$ and $q_{Z}:(Z, f) \rightarrow(X, \tau)$ is right $\epsilon$-resolving, it follows that $d_{Z}\left(p_{Z}^{-1}\left(x_{j}\right), p_{Z}^{-1}\left(x_{j}^{\prime}\right)\right) \leq \epsilon$ for all $j \in \mathbf{Z}$. This implies that $x_{j}=x_{j}^{\prime}$ for all $j \in \mathbf{Z}$, because $p_{Z}$ is $\epsilon$-preserving and $\tau$ is $\epsilon$-expansive.

The following result shows, for an expansive homeomorphism $\tau: X \rightarrow X$ of a 0 -dimensional space, where to find the essentially LR endomorphisms of $(X, \tau)$. We do not know whether it can be extended to the case when $X$ is of dimension greater than 0 .

Proposition 4.10. Let $\tau: X \rightarrow X$ be an expansive homeomorphism of a 0dimensional space, and $\varphi$ an onto endomorphism of $(X, \tau)$. Then $\varphi \tau^{n}$ is an essentially LR endomorphism of $(X, \tau)$ for some $n \geq 0$ if and only if $\varphi$ is right closing.

Before we prove this proposition, we give some basic information on symbolic dynamics. For more details see [N1], [N2], or [LM].

Let $A$ be an alphabet (finite set of symbols). We define a metric $d$ on $A^{\mathbf{Z}}$ as follows. For $x=\left(a_{j}\right)_{j \in \mathbf{Z}}$ and $y=\left(b_{j}\right)_{j \in \mathbf{Z}}$ in $A^{\mathbf{Z}}$, if $x=y$, then $d(x, y)=0$, and otherwise $d(x, y)=1 /(1+k)$, where $k=\min \left\{|j| \mid a_{j} \neq b_{j}\right\}$. With this metric, $A^{\mathbf{Z}}$ is compact. We define a homeomorphism $\sigma_{A}: A^{\mathbf{Z}} \rightarrow A^{\mathbf{Z}}$ by $\sigma_{A}\left(\left(a_{j}\right)_{j \in \mathbf{Z}}\right)=$ $\left(a_{j+1}\right)_{j \in \mathbf{Z}},\left(a_{j}\right)_{j \in \mathbf{Z}} \in A^{\mathbf{Z}}$. If $Y \subset A^{\mathbf{Z}}$ is closed with $\sigma_{A}(Y)=Y$ and if $\sigma: Y \rightarrow Y$ is the restriction of $\sigma_{A}$ on $Y$, then the dynamical system $(Y, \sigma)$ is called a subshift over $A$.

Let $(Y, \sigma)$ be a subshift. For any $x=\left(a_{j}\right)_{j \in \mathbf{Z}} \in Y$, let $s_{Y}(x)=\left(a_{j}\right)_{j \geq 0}$. Let $\bar{Y}=\left\{s_{Y}(x) \mid x \in Y\right\}$ and let $\bar{\sigma}: \bar{Y} \rightarrow \bar{Y}$ be defined by $\bar{\sigma}\left(s_{Y}(x)\right)=s_{Y}(\sigma(x))$, $x \in Y$. Let $\bar{Y}$ be endowed with the metric $d_{\bar{Y}}$ such that for $\bar{x}=\left(a_{j}\right)_{j \geq 0}$ and $\bar{y} \in\left(b_{j}\right)_{j \geq 0}$ in $\bar{Y}, d_{\bar{Y}}(\bar{x}, \bar{y})=0$ if $\bar{x}=\bar{y}$, and otherwise $d_{\bar{Y}}(\bar{x}, \bar{y})=1 /(1+k)$, where $k=\min \left\{j \geq 0 \mid a_{j} \neq b_{j}\right\}$. Then $s_{Y}: Y \rightarrow \bar{Y}$ is a factor map of $(Y, \sigma)$ onto $(\bar{Y}, \bar{\sigma})$. We call $(\bar{Y}, \bar{\sigma})$ the one-sided subshift induced by $(Y, \sigma)$, or a one-sided subshift. Clearly $\sigma$ is $(1 / 2)$-expansive and $\bar{\sigma}$ is positively $(1 / 2)$-expansive. For an onto map $\tau: X \rightarrow X$ of a 0 -dimensional space, if $\tau$ is an expansive homeomorphism, then $(X, \tau)$ is conjugate to some subshift, and if $\tau$ is positively expansive, then $(X, \tau)$ is conjugate to some one-sided subshift (Hedlund $[\mathrm{H}]$, Reddy $[\mathrm{R}]$ ).

Let $(Y, \sigma)$ be a subshift over an alphabet $A$. For $n \in \mathbf{N}$, let $L_{n}(Y)$ denote the set of all $n$-blocks or words of length $n$ that appear on some point in $Y$, i.e.,

$$
L_{n}(Y)=\left\{a_{0} \cdots a_{n-1} \mid\left(a_{j}\right)_{j \in \mathbf{Z}} \in Y, a_{j} \in A\right\} .
$$

Let $(Y, \sigma)$ and $\left(Y^{\prime}, \sigma^{\prime}\right)$ be subshifts. Let $m$ and $n$ be nonnegative integers. A mapping $\phi: Y \rightarrow Y^{\prime}$ is called a block map of $(m, n)$ type if it is given by a mapping $\Phi: L_{m+n+1}(Y) \rightarrow L_{1}\left(Y^{\prime}\right)$ in such a way that, for $\left(a_{j}\right)_{j \in \mathbf{Z}} \in Y$ with $a_{j} \in L_{1}(Y)$, $\phi\left(\left(a_{j}\right)_{j \in \mathbf{Z}}\right)=\left(b_{j}\right)_{j \in \mathbf{Z}}$ with $b_{j}=\Phi\left(a_{-m+j} \cdots a_{n+j}\right), j \in \mathbf{Z}$. In particular, a block map of $(0,0)$ type is called a 1-block map. A mapping $\phi: Y \rightarrow Y^{\prime}$ is a factor map of $(Y, \sigma)$ into $\left(Y^{\prime}, \sigma^{\prime}\right)$ if and only if $\phi$ is a block map of $(m, n)$ type for some $m, n \geq 0$, and a mapping $\bar{\phi}: \bar{Y} \rightarrow \bar{Y}^{\prime}$ is a factor map of $(\bar{Y}, \bar{\sigma})$ into $\left(\bar{Y}^{\prime}, \bar{\sigma}^{\prime}\right)$ if and only if for some $n \geq 0$ there is a block map $\phi: Y \rightarrow Y^{\prime}$ of $(0, n)$ type such that $\bar{\phi} s_{Y}=s_{Y^{\prime}} \phi$ (Curtis, Hedlund, and Lyndon [H]). If $\bar{\phi} s_{Y}=s_{Y^{\prime}} \phi$, then $\bar{\phi}$ is said to be induced by $\phi$.

Let $k \in \mathbf{N}$. We define the higher block system of order $k$ of a subshift $(Y, \sigma)$ to be the subshift $\left(Y^{[k]}, \sigma^{[k]}\right)$ over $L_{k}(Y)$ such that

$$
Y^{[k]}=\left\{\left(a_{j} \cdots a_{j+k-1}\right)_{j \in \mathbf{Z}} \mid\left(a_{j}\right)_{j \in \mathbf{Z}} \in Y, a_{j} \in L_{1}(Y)\right\} .
$$


We also define the higher block system of order $k$ of a one-sided subshift $(\bar{Y}, \bar{\sigma})$ to be the one-sided subshift $\left(\bar{Y}^{[k]}, \bar{\sigma}^{[k]}\right)$ over $L_{k}(Y)$ such that $\bar{Y}^{[k]}=s_{Y^{[k]}}\left(Y^{[k]}\right)$. Let $\rho_{Y, k}: Y \rightarrow Y^{[k]}$ be defined by $\rho_{Y, k}\left(\left(a_{j}\right)_{j \in \mathbf{Z}}\right)=\left(a_{j} \cdots a_{j+k-1}\right)_{j \in \mathbf{Z}},\left(a_{j}\right)_{j \in \mathbf{Z}} \in Y$. Then $\rho_{Y, k}$ is a conjugacy of $(Y, \sigma)$ onto $\left(Y^{[k]}, \sigma^{[k]}\right)$ and a block map of $(0, k)$ type, and $\rho_{Y, k}^{-1}$ is a 1-block map. Clearly $\bar{\rho}_{Y, k}$ is a conjugacy of $(\bar{Y}, \bar{\sigma})$ onto $\left(\bar{Y}^{[k]}, \bar{\sigma}^{[k]}\right)$. If $\phi:(Y, \sigma) \rightarrow\left(Y^{\prime}, \sigma^{\prime}\right)$ is a factor map between subshifts, we let $\phi^{[k]}$ denote the factor map of $\left(Y^{[k]}, \sigma^{[k]}\right)$ into $\left(Y^{\prime[k]}, \sigma^{\prime[k]}\right)$ such that if $\phi\left(\left(a_{j}\right)_{j \in \mathbf{Z}}\right)=\left(b_{j}\right)_{j \in \mathbf{Z}},\left(a_{j}\right)_{j \in \mathbf{Z}} \in$ $Y, a_{j} \in L_{1}(Y)$, then $\phi^{[k]}\left(\left(a_{j} \cdots a_{j+k-1}\right)_{j \in \mathbf{Z}}\right)=\left(b_{j} \cdots b_{j+k-1}\right)_{j \in \mathbf{Z}}$. It is clear that $\rho_{Y^{\prime}, k} \phi=\phi^{[k]} \rho_{Y, k}$.

Let $G$ be a graph. (A graph means a directed graph which may have multiple arcs and multiple loops.) Let $A_{G}$ and $V_{G}$ denote the arc-set of $G$ and the vertex-set of $G$, respectively. Let $i_{G}: A_{G} \rightarrow V_{G}$ and $t_{G}: A_{G} \rightarrow V_{G}$ be the mappings such that, for $a \in A_{G}, i_{G}(a)$ and $t_{G}(a)$ are the initial and terminal vertices of $a$, respectively. Let

$$
Y_{G}=\left\{\left(a_{j}\right)_{j \in \mathbf{Z}} \mid \forall j \in \mathbf{Z}, a_{j} \in A_{G}, t_{G}\left(a_{j}\right)=i_{G}\left(a_{j+1}\right)\right\} .
$$

Then we have a subshift $\left(Y_{G}, \sigma_{G}\right)$ over $A_{G}$, which is called the topological Markov shift defined by $G$. For a subshift $(Y, \sigma), \sigma$ has $\operatorname{POTP}$ if and only if $(Y, \sigma)$ is conjugate to a topological Markov shift (Walters [W]).

Proof of Proposition 4.10. The only-if part follows from Remark 4.9. Hence by the above it suffices to prove that if $\varphi$ is a right closing endomorphism of a subshift $(Y, \sigma)$, there is $N \in \mathbf{N}$ such that $\varphi \sigma^{N}$ is an essentially LR endomorphism of $(Y, \sigma)$. Let $\varphi$ be a right closing endomorphism of a subshift $(Y, \sigma)$. We may assume that $\varphi$ is a block map of $(0, k)$ type with $k \geq 0$. Let $\Phi: L_{k+1}(Y) \rightarrow L_{1}(Y)$ be a mapping which gives $\varphi$. For convenience we extend $\Phi$ to $\Phi: \bigcup_{l \in \mathbf{N}} L_{k+l}(Y) \rightarrow \bigcup_{l \in \mathbf{N}} L_{l}(Y)$ as follows: for $a_{1} \cdots a_{k+l} \in L_{k+l}(Y), a_{j} \in L_{1}(Y)$,

$$
\Phi\left(a_{1} \cdots a_{k+l}\right)=\Phi\left(a_{1} \cdots a_{k+1}\right) \Phi\left(a_{2} \cdots a_{k+2}\right) \cdots \Phi\left(a_{l} \cdots a_{k+l}\right) .
$$

Since $\varphi$ is right closing, it follows from a standard compactness argument that there is an integer $r>k / 2$ such that there is no pair of points $\left(a_{j}\right)_{j \in \mathbf{Z}}$ and $\left(b_{j}\right)_{j \in \mathbf{Z}}$ in $Y$ with

$$
a_{-r} \cdots a_{-1}=b_{-r} \cdots b_{-1}, \quad a_{0} \neq b_{0}, \quad \text { and } \Phi\left(a_{-r} \cdots a_{r}\right)=\Phi\left(b_{-r} \cdots b_{r}\right) .
$$

Let $s=2 r+1$. Let $B=L_{s-k}(Y)$ and let $C=\left\{\Phi(\alpha) \mid \alpha \in L_{s}(Y)\right\}$. Let $G$ be the graph such that $A_{G}=L_{s}(Y), V_{G}=L_{s-1}(Y)$, and for $\alpha=a_{1} \cdots a_{s}$ with $a_{j} \in L_{1}(Y), i_{G}(\alpha)=a_{1} \cdots a_{s-1}$ and $t_{G}(\alpha)=a_{2} \cdots a_{s}$. Let $\Phi^{\prime}: A_{G} \rightarrow C$ be defined by $\Phi^{\prime}(\alpha)=\Phi(\alpha), \alpha \in A_{G}$. Let $\phi:\left(Y_{G}, \sigma_{G}\right) \rightarrow\left(C^{\mathbf{Z}}, \sigma_{C}\right)$ be the 1-block map given by $\Phi^{\prime}$. We shall see that $\phi$ is right closing.

Suppose that there are distinct $\left(\alpha_{j}\right)_{j \in \mathbf{Z}},\left(\beta_{j}\right)_{j \in \mathbf{Z}} \in Y_{G}, \alpha_{j}, \beta_{j} \in A_{G}$, such that $\Phi^{\prime}\left(\alpha_{j}\right)=\Phi^{\prime}\left(\beta_{j}\right)$ for $j \in \mathbf{Z}$ and $\lim _{n \rightarrow \infty} d_{Y_{G}}\left(\sigma_{G}^{-n}\left(\left(\alpha_{j}\right)_{j \in \mathbf{Z}}\right), \sigma_{G}^{-n}\left(\left(\beta_{j}\right)_{j \in \mathbf{Z}}\right)\right)=0$. This and a standard compactness argument show that there is $J \in \mathbf{Z}$ such that $\alpha_{j}=\beta_{j}$ for $j<J$. Since $\left(\alpha_{j}\right)_{j \in \mathbf{Z}} \neq\left(\beta_{j}\right)_{j \in \mathbf{Z}}$, we may assume that $\alpha_{J} \neq \beta_{J}$. Put $\alpha_{j}=a_{-r}^{(j)} \cdots a_{r}^{(j)}$ and $\beta_{j}=b_{-r}^{(j)} \cdots b_{r}^{(j)}$, with $a_{i}^{(j)}, b_{i}^{(j)} \in L_{1}(Y)$ for $j \in \mathbf{Z}$. Then $a_{-r}^{(J)} \cdots a_{r-1}^{(J)}=b_{-r}^{(J)} \cdots b_{r-1}^{(J)}$ and $a_{r}^{(J)} \neq b_{r}^{(J)}$. Since $\left(\alpha_{j}\right)_{j \in \mathbf{Z}},\left(\beta_{j}\right)_{j \in \mathbf{Z}} \in Y_{G}$, it follows that $a_{-r}^{(J+r)} \cdots a_{-1}^{(J+r)}=a_{0}^{(J)} \cdots a_{r-1}^{(J)}=b_{0}^{(J)} \cdots b_{r-1}^{(J)}=b_{-r}^{(J+r)} \cdots b_{-1}^{(J+r)}$ and $a_{0}^{(J+r)}=$ $a_{r}^{(J)} \neq b_{r}^{(J)}=b_{0}^{(J+r)}$. These contradict the property of $r$, since $a_{-r}^{(J+r)} \cdots a_{r}^{(J+r)}$ and $b_{-r}^{(J+r)} \cdots b_{r}^{(J+r)}$ are in $L_{2 r+1}(Y)$ and $\Phi\left(a_{-r}^{(J+r)} \cdots a_{r}^{(J+r)}\right)=\Phi\left(b_{-r}^{(J+r)} \cdots b_{r}^{(J+r)}\right)$. 
By using the fact that $\phi$ is right closing and by modifying straightforwardly the proof of Proposition 6.30 of [N1] (consider the 'induced right resolving $\lambda$-graph' of the 'higher block $\lambda$-graph' $\mathcal{G}^{[m]}$ of order $m$ of the ' $\lambda$-graph' $\mathcal{G}=(G, \lambda)$ with $\lambda=\Phi^{\prime}$ for $m \in \mathbf{N}$ ), it is proved that there are an integer $N \geq 0$, a graph $\Gamma$, and a pair of mappings $p: A_{\Gamma} \rightarrow B$ and $q: A_{\Gamma} \rightarrow C$ having the following properties: (i) for any $\gamma, \gamma^{\prime} \in A_{\Gamma}$, if $t_{\Gamma}(\gamma)=t_{\Gamma}\left(\gamma^{\prime}\right)$ and $p(\gamma)=p\left(\gamma^{\prime}\right)$, then $\gamma=\gamma^{\prime}$; (ii) for any $\gamma, \gamma^{\prime} \in A_{\Gamma}$, if $i_{\Gamma}(\gamma)=i_{\Gamma}\left(\gamma^{\prime}\right)$ and $q(\gamma)=q\left(\gamma^{\prime}\right)$, then $\gamma=\gamma^{\prime}$; (iii) if $\xi:\left(Y_{\Gamma}, \sigma_{\Gamma}\right) \rightarrow\left(B^{\mathbf{Z}}, \sigma_{B}\right)$ and $\eta:\left(Y_{\Gamma}, \sigma_{\Gamma}\right) \rightarrow\left(C^{\mathbf{Z}}, \sigma_{C}\right)$ are the 1-block maps given by $p$ and $q$, respectively, then $\xi$ is $1-1$, and $\eta \xi^{-1} \mid \xi\left(Y_{\Gamma}\right): \xi\left(Y_{\Gamma}\right) \rightarrow \eta\left(Y_{\Gamma}\right)$ is an extension of $\varphi^{[s-k]}\left(\sigma^{[s-k]}\right)^{N}$.

Let $W=Y^{[s-k]}$, let $U=\left(Y^{[s-k]}\right)^{[2]}$, let $V=\xi^{-1}\left(Y^{[s-k]}\right)$, and let $i_{U}: U \rightarrow W$ and $t_{U}: U \rightarrow W$ be defined by

$$
\begin{aligned}
i_{U}\left(\left(\hat{b}_{j} \hat{b}_{j+1}\right)_{j \in \mathbf{Z}}\right)=\left(\hat{b}_{j}\right)_{j \in \mathbf{Z}} \text { and } t_{U}\left(\left(\hat{b}_{j} \hat{b}_{j+1}\right)_{j \in \mathbf{Z}}\right)=\left(\hat{b}_{j+1}\right)_{j \in \mathbf{Z}}, & \left(\hat{b}_{j}\right)_{j \in \mathbf{Z}} \in Y^{[s-k]}, \hat{b}_{j} \in B,
\end{aligned}
$$

and let $p_{V}: V \rightarrow W$ and $q_{V}: V \rightarrow W$ be defined by

$$
p_{V}=\xi \mid\left(\xi^{-1}\left(Y^{[s-k]}\right)\right) \text { and } q_{V}=\eta \mid\left(\xi^{-1}\left(Y^{[s-k]}\right)\right) \text {. }
$$

Then it is straightforward to check, using (i), (ii), and (iii), that $T=\left(i_{U}, t_{U}: U \rightarrow\right.$ $\left.W ; p_{V}, q_{V}: V \rightarrow W\right)$ is an LR $(1 / 2)$-textile-orbit-system, $i_{U}^{-1} t_{U}\left(=\left(\sigma^{[s-k]}\right)^{[2]}=\right.$ $\left.\sigma^{[s-k+1]}\right)$ is $(1 / 2)$-expansive, and

$$
q_{Z} p_{Z}^{-1}=\left(\varphi^{[s-k]}\left(\sigma^{[s-k]}\right)^{N}\right)^{[2]}=\left(\varphi \sigma^{N}\right)^{[s-k+1]}
$$

where $Z$ is the square space of $T$. Therefore $\left(\varphi \sigma^{N}\right)^{[l]}$ is an LR endomorphism of $\left(Y^{[l]}, \sigma^{[l]}\right)$ with $l=s-k+1$. Thus $\varphi \sigma^{N}$ is an essentially LR endomorphism of $(X, \sigma)$.

Corollary 4.11. Let $\tau: X \rightarrow X$ be a positively expansive onto map of a 0dimensional space, and $\varphi$ an onto endomorphism of $(X, \tau)$. Then $\varphi \tau^{n}$ is an essentially positively LR endomorphism of $(X, \tau)$ for some $n \geq 0$ if and only if $\varphi$ is right closing.

Proof. By Remark 4.9, the only-if part holds. Assume that $\varphi$ is right closing. Then there are a subshift $(Y, \sigma)$, an endomorphism $f$ of $(Y, \sigma)$ which is a block map of $(0, k)$ type for some $k \geq 0$, and a conjugacy $\psi:(X, \tau) \rightarrow(\bar{Y}, \bar{\sigma})$ such that $\psi \varphi \psi^{-1}=\bar{f}$, where $(\bar{Y}, \bar{\sigma})$ is the one-sided subshift induced by $(Y, \sigma)$ and $\bar{f}$ is the endomorphism of $(\bar{Y}, \bar{\sigma})$ induced by $f$. Since $\bar{f}$ is right closing, so is $f$. Let $T=$ $\left(i_{U}, t_{U}: U \rightarrow W ; p_{V}, q_{V}: V \rightarrow W\right)$ be an LR (1/2)-textile-orbit-system constructed in the same way as in the proof of Proposition 4.10 for $f$. Let $N$ and $l$ be defined in the same way as there for $f$. Let $\bar{T}=\left(i_{\bar{U}}, t_{\bar{U}}: \bar{U} \rightarrow \bar{W} ; p_{\bar{V}}, q_{\bar{V}}: \bar{V} \rightarrow \bar{W}\right)$, where $\bar{U}, \bar{V}, \bar{W}$ are the one-sided subshift spaces induced by the subshift spaces $U, V, W$, respectively and $i_{\bar{U}}, t_{\bar{U}}, p_{\bar{V}}, q_{\bar{V}}$ are respectively $\bar{i}_{U}, \bar{t}_{U}, \bar{p}_{V}, \bar{q}_{V}$ induced by the 1-block maps $i_{U}, t_{U}, p_{V}, q_{V}$, respectively. Clearly $i_{\bar{U}}$ is $1-1$, and so is $p_{\bar{V}}$ because for $\xi$ in (iii) of the proof of Proposition 4.10 it can be seen that $\xi^{-1} \mid \xi\left(Y_{\Gamma}\right): \xi\left(Y_{\Gamma}\right) \rightarrow Y_{\Gamma}$ is a block map of $(0, m)$ type for some $m \geq 0$. Thus $\bar{T}$ is an LR (1/2)-textile-orbitsystem with $i_{\bar{U}}^{-1} t_{\bar{U}}=\bar{\sigma}^{[l]}$ and $q_{\bar{Z}} p_{\bar{Z}}^{-1}=\left(\overline{\bar{f}} \bar{\sigma}^{N}\right)^{[l]}$, where $\bar{Z}$ is the square space of $\bar{T}$. Therefore $\left(\bar{f} \bar{\sigma}^{N}\right)^{[l]}$ is a positively LR endomorphism of $\left(\bar{Y}^{[l]}, \bar{\sigma}^{[l]}\right)$, so that $\varphi \tau^{N}$ is an essentially positively LR endomorphism of $(X, \tau)$. 


\section{REFERENCES}

[AH] N. Aoki and K. Hiraide, Topological Theory of Dynamical Systems, North-Holland, Amsterdam, 1994. MR 95m:58095

[AS] N. Aoki and K. Shiraiwa, Dynamical Systems and Entropy, Kyoritsu Shuppan, Tokyo, 1985 (in Japanese).

[BM] F. Blanchard and A. Maass, Dynamical properties of expansive one-sided cellular automata, Israel J. Math. 99 (1997), 149-174. MR 98g:58089

[Bow1] R. Bowen, Topological entropy and Axiom A, Proc. Sympos. Pure Math. 14, Amer. Math. Soc., Providence, R. I., 1970, pp. 23-41. MR 41:7066

[Bow2] - Markov partitions for Axiom A diffeomorphisms, Amer. J. Math. 92 (1970), 725-747. MR 43:2740

[Bow3] - Periodic points and measures for Axiom A diffeomorphisms, Trans. Amer. Math. Soc. 154 (1971), 377-397. MR 43:8084

[Bow4] _ Equilibrium States and the Ergodic Theory of Anosov Diffeomorphisms, Lecture Notes in Math. 470, Springer-Verlag, 1975. MR 56:1364

[Bow5] _ On Axiom A Diffeomorphisms, CBMS Regional Conf. Ser. in Math. 35, Amer. Math. Soc., Providence R. I., 1978. MR 58:2888

[Boy] M. Boyle, Factoring factor maps, J. London Math. Soc. (2) 57 (1998), 491-502. MR 99i: 58048

[BFF] M. Boyle, D. Fiebig and U. Fiebig, A dimension group for local homeomorphisms and endomorphisms of one-sided shifts of finite type, J. Reine Angew. Math. 487 (1997), 27-59. MR 98i:54020

[BK] M. Boyle and W. Krieger, Periodic points and automorphisms of the shift, Trans. Amer. Math. Soc. 302 (1987), 125-149. MR 88g:54065

[BL] M. Boyle and D. Lind, Expansive subdynamics, Trans. Amer. Math. Soc. 349 (1997), 55-102. MR 97d:58115

[BoMa] M. Boyle and A. Maass, Expansive invertible onesided cellular automata, to appear in J. Math. Soc. Japan.

[CP] E. Coven and M. Paul, Endomorphisms of irreducible shifts of finite type, Math. Systems Theory 8 (1974), 167-175. MR 52:4259

[F] D. Fiebig, private communication, 1996.

[Fr] D. Fried, Finitely presented dynamical systems, Ergodic Theory Dynam. Systems 7 (1987), 489-507. MR 89h:58157

[H] G. A. Hedlund, Endomorphisms and automorphisms of the shift dynamical system, Math. Systems Theory 3 (1969), 320-375. MR 41:4510

[Hi] K. Hiraide, Dynamical systems of expansive maps on compact manifolds, Sugaku Expositions 5 (1992), 133-154. MR 91d:58197 (Japanese original)

[K] P. Kůrka, Languages, equicontinuity and attractors in cellular automata, Ergodic Theory Dynam. Systems 17 (1997), 417-433. MR 98b:58092

[LM] D. Lind and B. Marcus, An Introduction to Symbolic Dynamics and Coding, Cambridge University Press, 1995. MR 97a:58050

[N1] M. Nasu, Textile systems for endomorphisms and automorphisms of the shift, Mem. Amer. Math. Soc. 546 (1995). MR 95i:54051

[N2] Maps in symbolic dynamics, in Lecture Notes of The Tenth KAIST Mathematics Workshop 1995, ed. G. H. Choe, Korea Advanced Institute of Science and Technology, Mathematics Research Center, Taejon, 1996.

[R] W. L. Reddy, Lifting expansive homeomorphisms to symbolic flows, Math. Systems Theory 2 (1968), 91-92. MR 36:7127

[S] S. Smale, Differentiable dynamical systems, Bull. Amer. Math. Soc. 73 (1967), 747-817. MR 37:3598

[W] P. Walters, On the pseudo-orbit tracing property and its relationship to stability, Lecture Notes in Math. 668, Springer-Verlag, 1978, 231-244. MR 80d:58055

Department of Applied Mathematics, Faculty of Engineering, Hiroshima University, Higashi-Hiroshima 739-8527, JaPAN.

E-mail address: nasu@amath.hiroshima-u.ac.jp 